Supplementary Information for

\title{
A Comprehensive Observational Based Multiphase Chemical Model Analysis of the Sulfur Dioxide Oxidations in both Summer and Winter
}

Huan Song ${ }^{1}$, Keding $\mathrm{Lu}^{1 *}$, Can Ye ${ }^{1}$, Huabin Dong ${ }^{1}$, Shule $\mathrm{Li}^{1}$, Shiyi Chen ${ }^{1}$, Zhijun $\mathrm{Wu}^{1}$, Mei Zheng ${ }^{1}$, Limin Zeng ${ }^{1}$, Min $\mathrm{Hu}^{1}$ \& Yuanhang Zhang ${ }^{1}$

${ }^{1}$ State Key Joint Laboratory or Environmental Simulation and Pollution Control, College of Environmental Sciences and Engineering, Peking University, Beijing, China

10 Correspondence to: Keding Lu (k.lu@pku.edu.cn)

This PDF file includes:

Supplementary text

Figures $\mathrm{S} 1$ to $\mathrm{S} 9$

Tables $\mathrm{S} 1$ to $\mathrm{S} 9$

15 SI References 


\section{Supplementary Information Text}

\section{Text S1. Activity coefficients of main reactants in the PKU-MARK model}

20 The properties of electrolytes play an important role in the kinetic salt effect in the aqueous phase reaction. Atmospheric heterogeneous reactions occurring in aerosol deliquescent particles are characterized by high ionic strength $\left(I_{s}\right)$. In these multicomponent mixture, reaction rates should be replaced by the activity coefficient, which representing the thermodynamic non-ideality caused by all-molecular interactions (Rusumdar et al., 2016;Rusumdar et al., 2020). Suitable multiphase chemistry models should apply activity coefficients instead of reaction rate constants in non-ideal solution. Heterogeneous processed in

25 clouds and haze may be considered as occurring in dilute electrolytes and there is no need to consider the influence of ionic strength, this is not the case for high ionic strength deliquescent particles. Based on the measurement of ambient aerosol deliquescent particles (Herrmann et al., 2015), in marine areas, the ionic strength is about $6 \mathrm{M}$ and in urban environments can reach about 8-18 M. Fountoukis and Nenes using the ISORROPIA-II model predicted high levels of ionic strength ranging between 13 and $43 \mathrm{M}$ during the severe Beijing Haze (Fountoukis and Nenes, 2007a). In some extreme cases, the ionic strength

30 of aerosol deliquescent particles can even reach $100 \mathrm{M}$ (Cheng et al., 2016a). In two field campaign mentioned in this study, the mean value of ionic strength is $56.55 \pm 39.83 \mathrm{M}( \pm 1 \sigma)$ in winter and $24.26 \pm 13.3 \mathrm{M}( \pm 1 \sigma)$ in summer in haze periods $\left(\mathrm{PM}_{2.5}>75 \mu \mathrm{g} / \mathrm{m}^{3}\right)$, and in extremely situation, $175.45 \mathrm{M}$ in winter and $96.41 \mathrm{M}$ in summer. In these cases, large errors can be introduced in the model calculation without considering the influence of ionic strength on aqueous phase reaction rate and heterogeneous mass transport.

35 Several studies are developed to evaluate the effects of ionic strength on the activity of aqueous phase ions and organic matters (Pitzer, 1991;Li et al., 1994;Polka et al., 1994;Ming and Russell, 2002;Raatikainen and Laaksonen, 2005;Clegg et al., 2008;Zuend et al., 2008;Zuend et al., 2011), and during the latest year considerable effort has been devoted to developing kinetic model frameworks for the modelling of processes in multicomponent atmospheric particles, which include both a detailed description of organic and inorganic multiphase chemistry as well as detailed thermodynamic comprehensions of its

40 non-ideal behavior (Rusumdar et al., 2016). In this study, the activity coefficients of TMI were calculated by the Extended Debye-Hückel equation (Ross and Noone, 1991;Linder and Murray, 1982;Kontogeorgis et al., 2018). Other ions and organic oxalic acid and its complexes used the typical values predicted by AIOMFAC model by Rusumdar (Rusumdar et al., 2020). As for neutral solutes which activity coefficients are not corrected in AIOMFAC model, such as $\mathrm{O}_{3}, \mathrm{O}_{2}$ and small molecules $\mathrm{OH}$ and $\mathrm{HO}_{2}$, logarithm of the activity coefficient of neutral solutes is a linear function of the effective ionic strength and the

45 Sechenov coefficient (Rischbieter et al., 2000;Beltran, 2003;Clever, 1983;Ross and Noone, 1991). Unfortunately, Setchenov parameters are unknown for $\mathrm{H}_{2} \mathrm{O}_{2}$, which is an important source of aqueous $\mathrm{OH}$ radical and other ROS. Several studies(Ali et al., 2014;Cheng et al., 2016b;Liu et al., 2020) showed that the formation rate of $\mathrm{S}(\mathrm{VI})$ by $\mathrm{H}_{2} \mathrm{O}_{2}$ increases with aerosol condensed phase solution ionic strength and proposed different expression with the limitation of maximal ionic strength equal 
to $5 \mathrm{M}$. In this case, considering the high value of ionic strength in the two field campaigns (averaged values are beyond $5 \mathrm{M}$ ), direct extrapolation of the observed/predicted $a-I$ relationship into such high ranges of ionic strength may not be appropriate. Thus, in this paper, the activity coefficient of $\mathrm{H}_{2} \mathrm{O}_{2}$ is only considered when calculating the sulfate formation rate. Corresponding typical activity coefficient values and calculation expressions are summarized in Table S2 and S3. The influence of ionic strength on gas phase molecular Henry's law coefficients were also considered in the MARK model which are summarized in Table S3.

\section{Text S2. The concentration of aerosol particle transition metals in urban areas}

For the lack of Mn concentration in $\mathrm{PM}_{2.5}$ during two field campaigns, we summarized the concentration of transition metals in urban areas, mainly in Beijing winter in Table S9. The mass concentration ratio of Fe/Mn is in the range of 8.6 to 31 in Beijing, and can up to 78 in India. In the calculation of sulfate formation, we used the mass concentration ratio of Fe/Mn as 28

60 which is a medium value of the ratios. The modeled ratio of soluble Fe (III) to total Fe in the whole winter field campaign was in the range of $0.02 \%$ to $27.63 \%$ with an average value of $1.63 \%$ and in the range of $0.04 \%$ to $3.29 \%$ with an average value of $0.79 \%$ in polluted and highly polluted conditions. The modeled ratio of soluble $\mathrm{Mn}$ (II) to total $\mathrm{Mn}$ in the whole winter field campaign was in the range of $0.01 \%$ to $97.21 \%$ with an average value of $21.78 \%$ and in the range of $0.01 \%$ to $80.46 \%$ with an average value of $19.83 \%$ in polluted and highly polluted conditions.

\section{Text S3. Four haze periods in PKU-17 observation}

Fig. S4 shows the diurnal trends of the key parameters measured in the PKU field campaign for four haze periods. All four haze periods lasted for 6 days. Due to the strict emission control policies enacted by the Chinese government, the concentration of $\mathrm{PM}_{2.5}$ decreased compared to the same period in 2016 while still cause severe haze pollution in $2017 . \mathrm{H}_{2} \mathrm{O}_{2}$ exhibited a typical diurnal pattern with a maximum in the afternoon and low concentrations in the morning and night. It was worth mentioning that some studies reported high $\mathrm{H}_{2} \mathrm{O}_{2}$ concentrations during haze episodes, while in our study the average concentration of $\mathrm{H}_{2} \mathrm{O}_{2}$ was only about $0.02 \mathrm{ppb}$. Low $\mathrm{OH}$ radicals and $\mathrm{O}_{3}$ concentrations indicated low photochemical activity. The largest $\mathrm{PM}_{2.5}$ concentrations of period IV and II were observed exceeding $150 \mu \mathrm{g} / \mathrm{m}^{3}$, which was coincident with higher concentrations of transition metal including $\mathrm{Fe}$ and $\mathrm{Cu}$. Period IV was characterized by lower gas-phase $\mathrm{H}_{2} \mathrm{O}_{2}$ and higher $\mathrm{RH}$ as well as higher aerosol liquid water content compared to the other three periods. Due to the lack of $\mathrm{OH}$ radical data during Period IV, averaged $\mathrm{OH}$ concentrations from the other three haze periods were used in the calculation causing small biases due to the reduced gas-phase oxidant pathway during the haze period. Period II was characterized with the highest $\mathrm{SO}_{2}$ concentration which was beneficial to the formation of secondary sulfate aerosol. The other two haze periods including Period I and Period III also own high levels of 24-hour averaged $\mathrm{PM}_{2.5}$ loading exceeding $75 \mu \mathrm{g} / \mathrm{m}^{3}$. However, according to the 
80 observed $\mathrm{SO}_{4}{ }^{2-}$ concentration, high concentrations of sulfate only appeared in the fourth stage of pollution indicating the importance of RH and aerosol TMI.

\section{Figures}

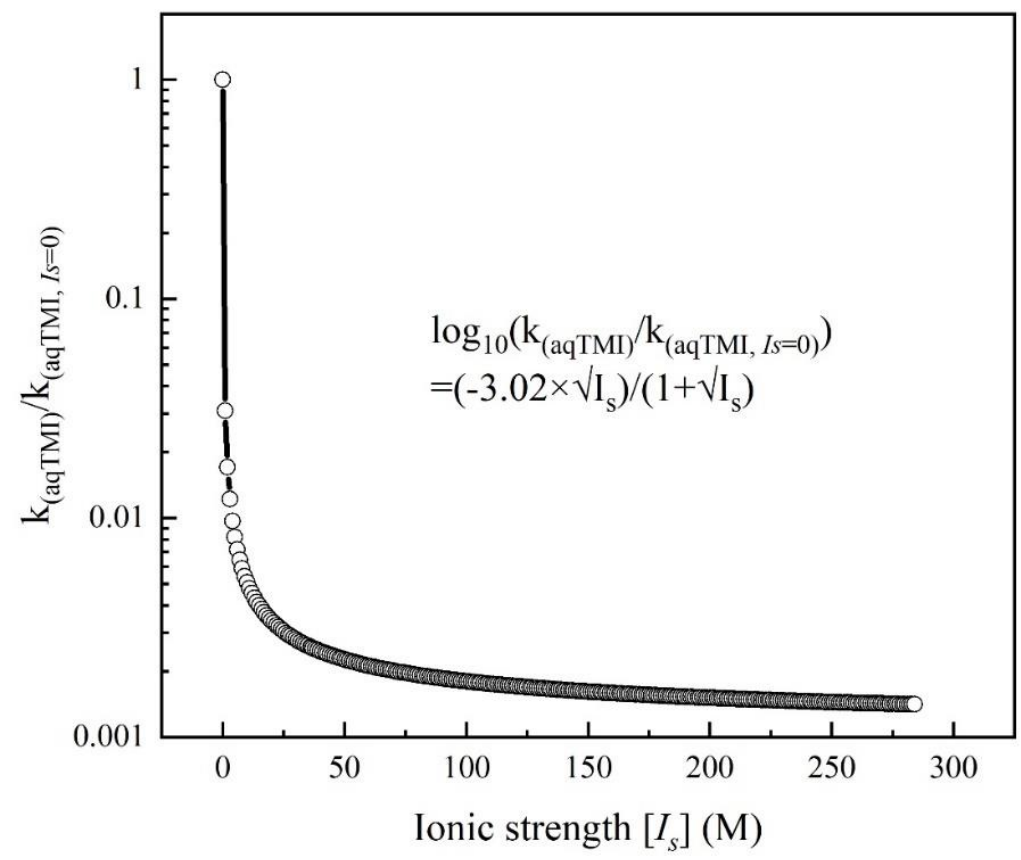

85 Fig. S1. Ionic strength of aerosol particle solution influence on the aqTMI rate constant. 

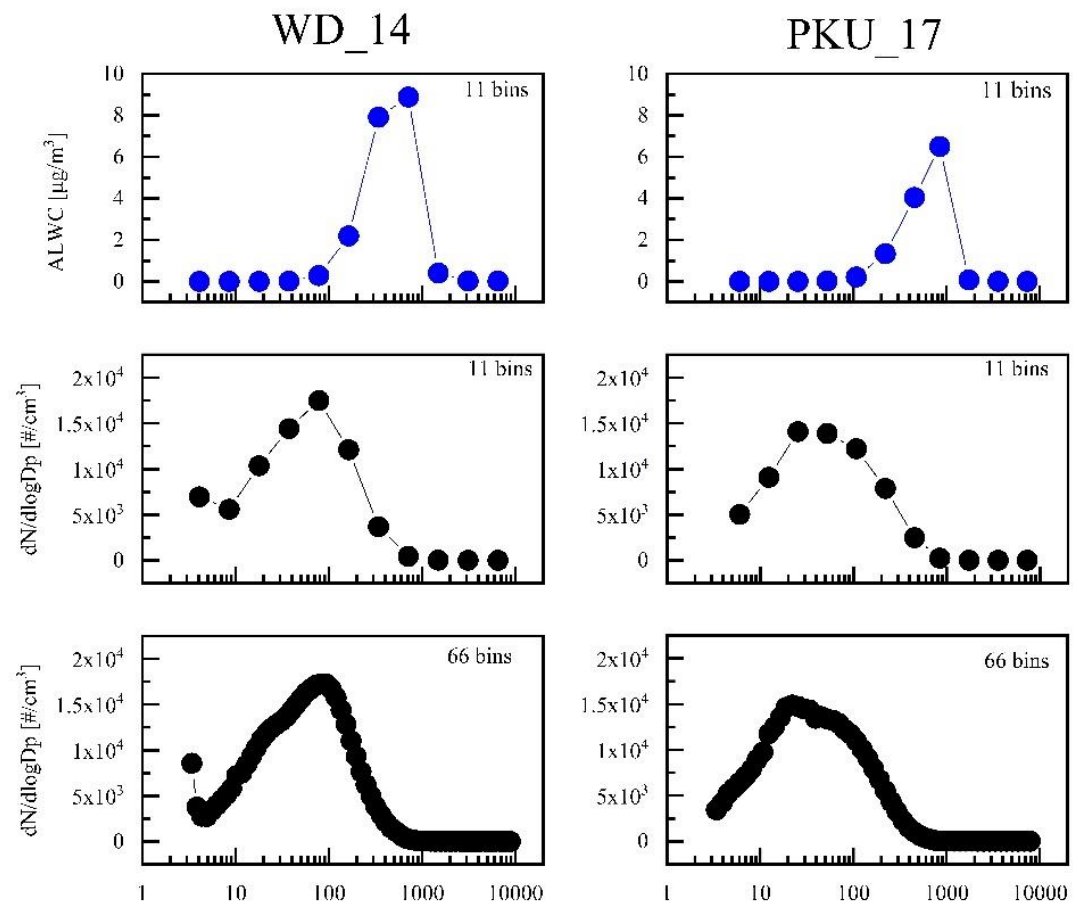

Fig. S2. Distribution of ALWC and number concentration with aerosol particle bins in two campaigns. 

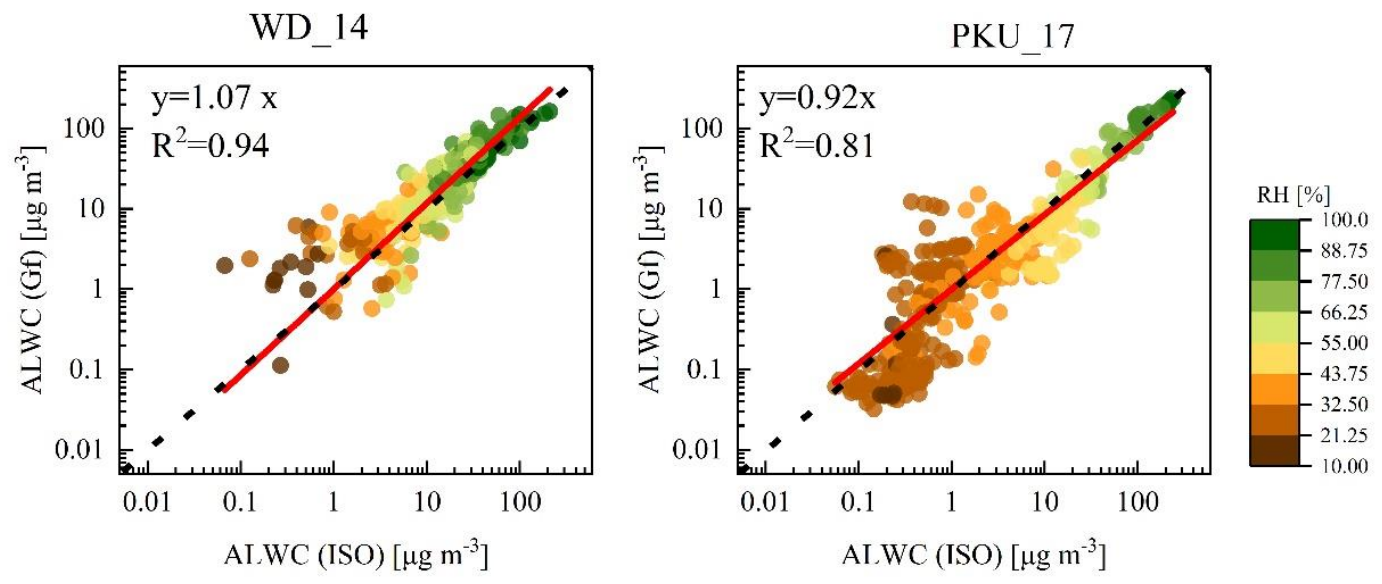

Fig. S3. Calculated aerosol water by ISORROPIA-II model and H-TDMA method in two field campaigns during haze periods. The plots were colored with the relative humidity values. The black dashed line in the figure is the 1:1 baseline, and the red solid line is the linear fitting result assuming the intercept is zero. 

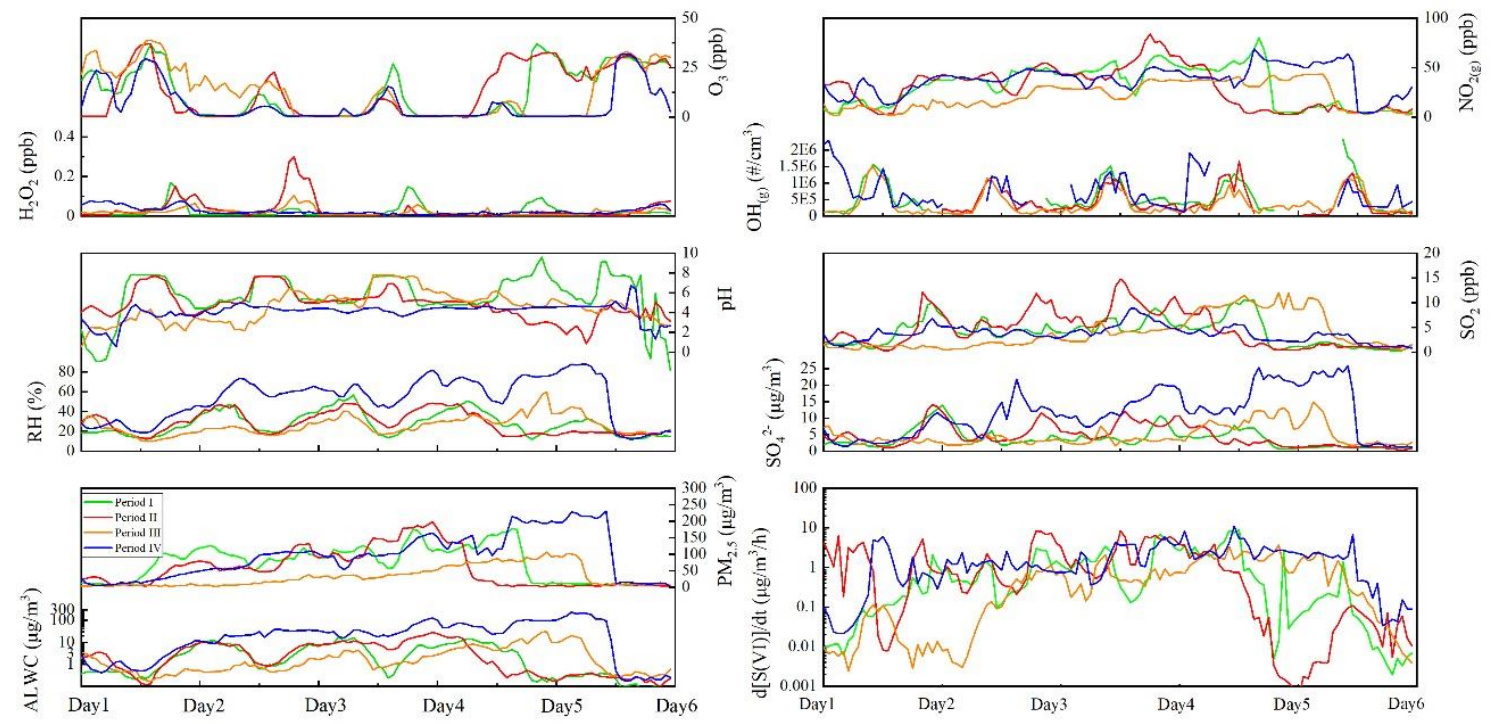

Fig. S4. Time series of observed gas-phase pollutants concentrations, RH, Temperature, $\mathrm{PM}_{2.5}$ mass loading and calculated aerosol $\mathrm{pH}$ and water content and sulfate formation rates in these four haze periods in PKU-17 field campaign. 


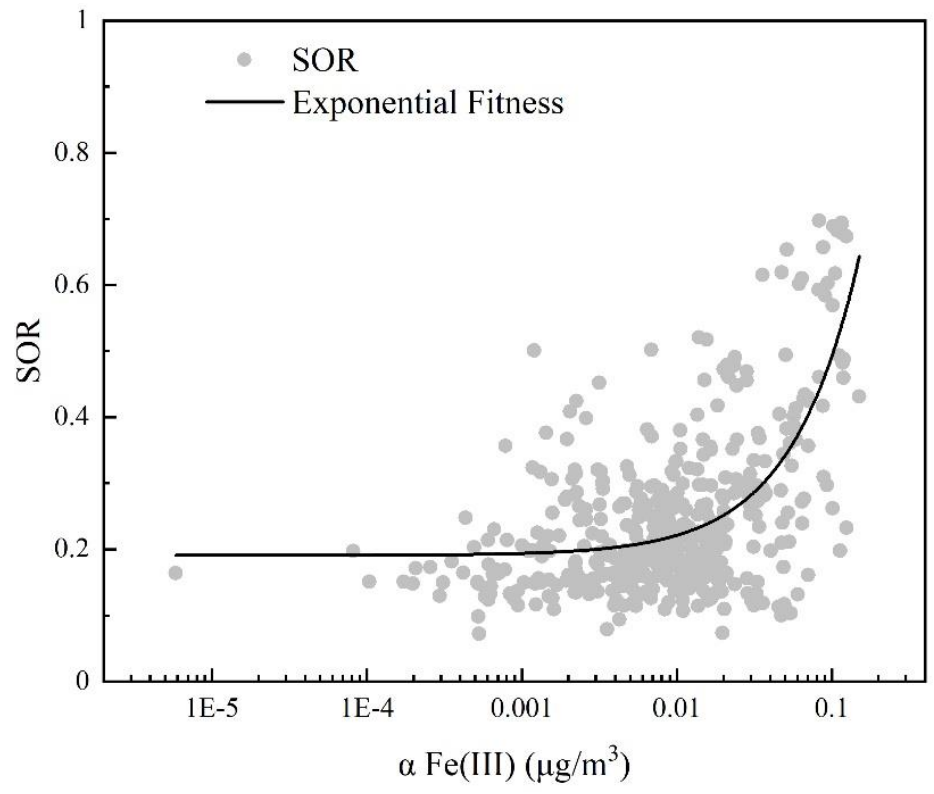

Fig. S5. SOR $\left(\equiv \mathrm{n}\left(\mathrm{SO}_{2}\right) / \mathrm{n}\left(\mathrm{SO}_{2}+\mathrm{SO}_{4}{ }^{2-}\right)\right)$ correlations with effective $\mathrm{Fe}$ (III) concentrations in PKU-17 winter field campaign.
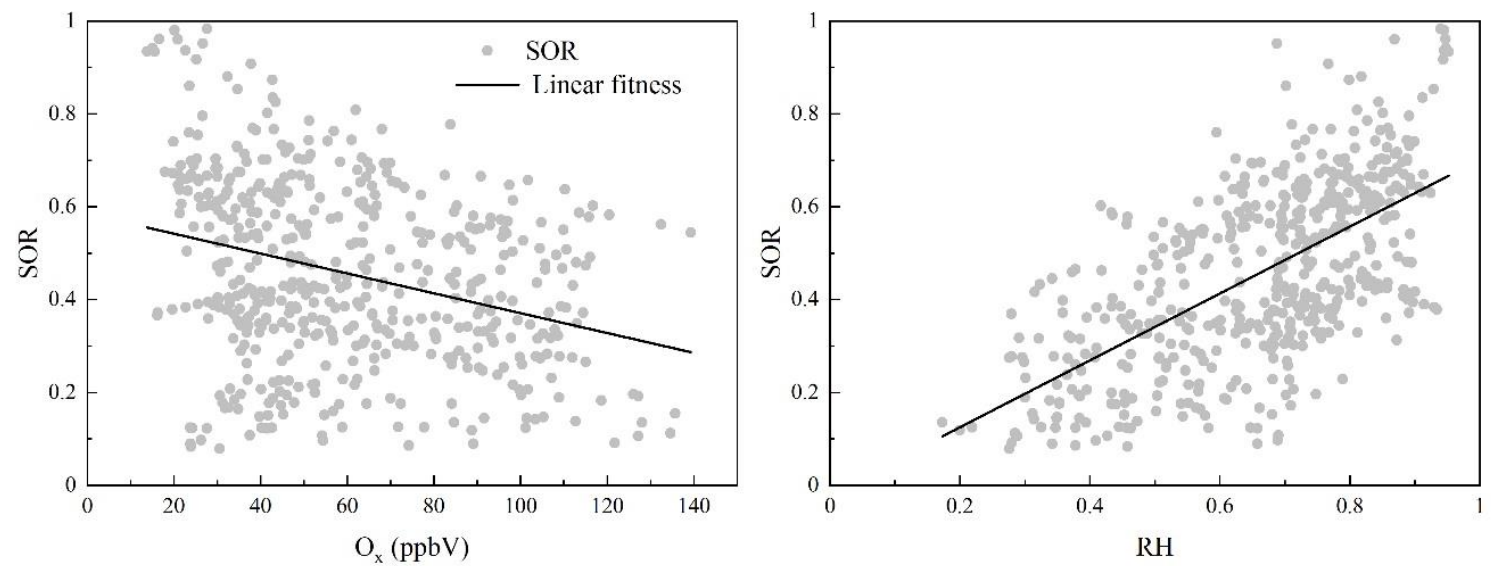

105 Fig. S6. SOR $\left(\equiv \mathrm{n}\left(\mathrm{SO}_{2}\right) / \mathrm{n}\left(\mathrm{SO}_{2}+\mathrm{SO}_{4}{ }^{2-}\right)\right)$ correlations with odd oxygen $\left(\left[\mathrm{O}_{\mathrm{x}}\right] \equiv\left[\mathrm{O}_{3}\right]+\left[\mathrm{NO}_{2}\right]\right)$ and relative humidity (RH) in WD14 summer field campaign. 


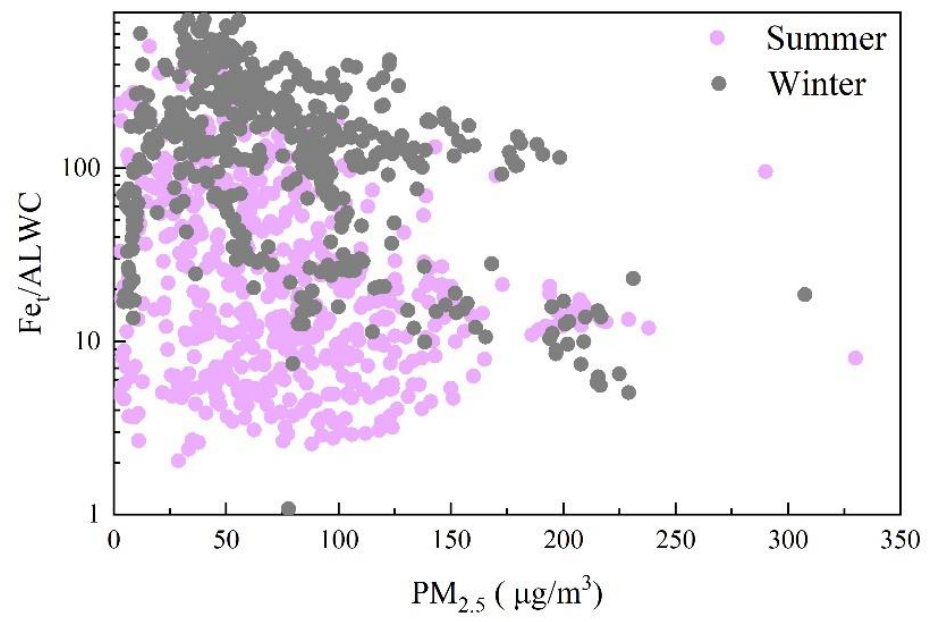

Fig. S7 The "dilution effect" of Fe mass concentration and ALWC increasing with PM mass in winter and summer.

110
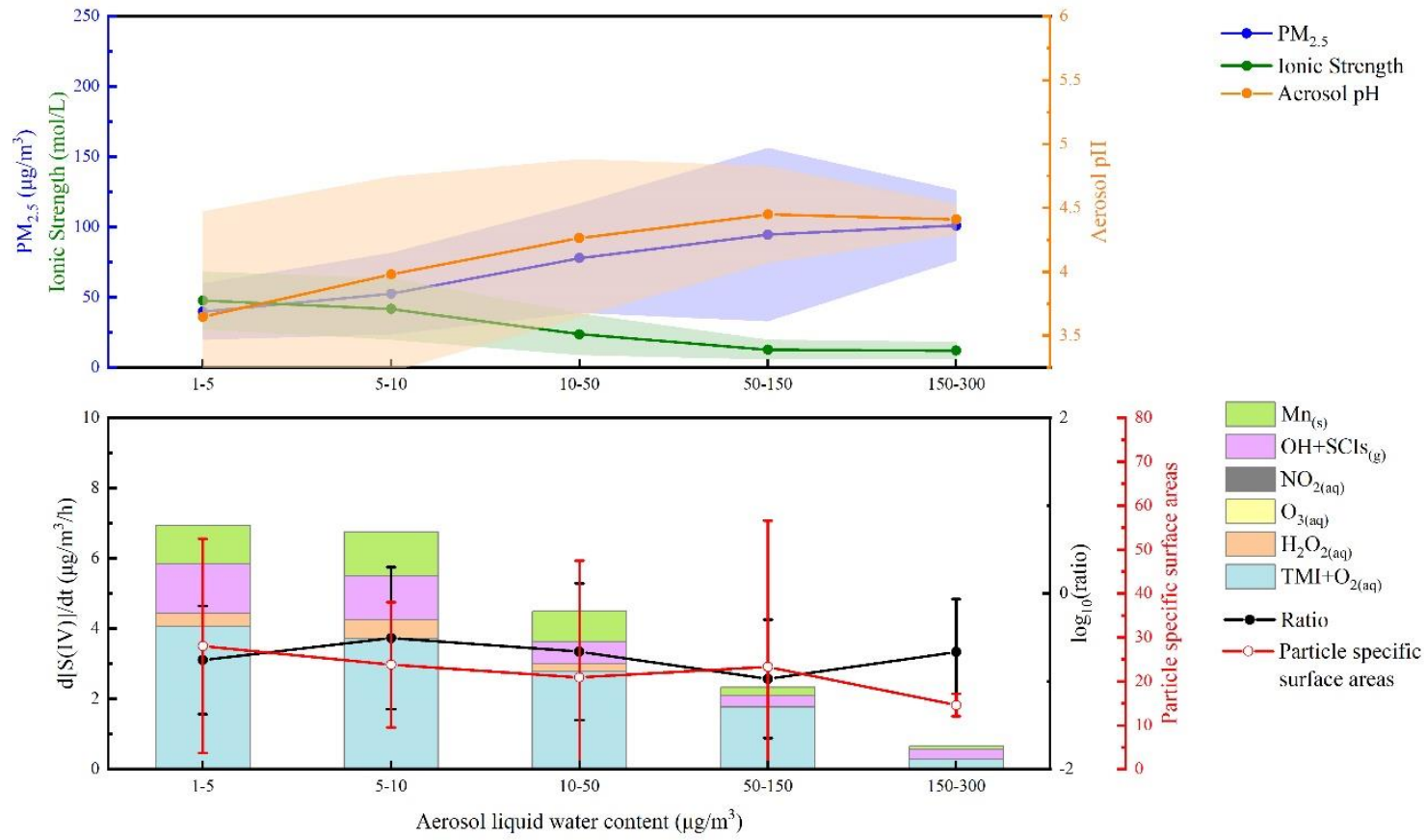

Fig. S8. Variation of $\mathrm{PM}_{2.5}$, ionic strength, aerosol $\mathrm{pH}$, particle specific surface areas and sulphate formation rates from different pathways with aerosol liquid water content (ALWC) during summer field campaign. The total number of valid data points shown in the figure is 501 . The shaded area refer to the error bar $( \pm 1 \sigma)$ of $\mathrm{PM}_{2.5}$ mass concentration, aerosol ionic

115 strength and $\mathrm{pH}$ calculated by ISORROPIA-II(Fountoukis and Nenes, 2007b). Ratio in the second panel refers to the ratio of contributions from Mn-surface and aqTMI to produce sulphate. Particle specific surface areas represent the ratio of particle surface area $\left(\mu \mathrm{m}^{2} / \mathrm{cm}^{3}\right)$ and mass concentration $\left(\mu \mathrm{g} / \mathrm{m}^{3}\right)$. 


\section{Tables}

120 Table S1. Reaction rate expression and constant for $\mathrm{SO} 2$ oxidation by $\mathrm{OH}$ in the gas-phase.

\begin{tabular}{lll}
\hline Oxidant & The reaction rate expression and constant ${ }^{\text {a }}$ & References \\
\hline $\mathrm{OH}$ & $R_{\mathrm{SO} 2+\mathrm{OH}}=k_{0}\left[\mathrm{SO}_{2}(\mathrm{~g})\right][\mathrm{OH}(\mathrm{g})]$ & Burkholder et al. (2020) \\
& $k_{\text {Olow }}=3.3 \times 10^{-31} \times(T / 300 \mathrm{~K})^{-4.3} \mathrm{~cm}^{6} \mathrm{~s}^{-1}$ & \\
& $k_{\text {0high }}=1.6 \times 10^{-12} \mathrm{~cm}^{3} \mathrm{~s}^{-1}$ & \\
& $F_{\mathrm{c}}=0.6$ &
\end{tabular}

${ }^{a}$ We report the low and the high pressure limit of $k$ for $\mathrm{SO}_{2}$ oxidation by OH. $F_{\mathrm{c}}$ is used to calculate the dependence of $k$ on pressure and temperature (details see the reference(Burkholder et al., 2020)). 
Table S2. Aqueous-phase reaction rate expressions, rate constants (k) and influence of ionic strength (Is) on $\mathrm{k}$ for sulfate 125 production in aerosol particle condensed phase.

\begin{tabular}{|c|c|c|c|}
\hline Oxidants & $\begin{array}{l}\text { The reaction rate expressions }\left(R_{\mathrm{S}(\mathrm{IV})+\mathrm{oxi}}\right), \text { constants }(k) \text { and } \\
\left.\text { influence of } I_{\mathrm{S}} \text { (in unit of } \mathrm{M}\right) \text { on } k^{\mathrm{a}}\end{array}$ & Notes & References \\
\hline \multirow[t]{8}{*}{$\mathrm{O}_{3}$} & $\left(k_{1}\left[\mathrm{H}_{2} \mathrm{SO}_{3}\right]+k_{2}\left[\mathrm{HSO}_{3}{ }^{-}\right]+k_{3}\left[\mathrm{SO}_{3}{ }^{2-}\right]\right)\left[\mathrm{O}_{3}(\mathrm{aq})\right]$ & & \multirow{4}{*}{$\begin{array}{l}\text { Seinfeld and Pandis } \\
\text { (2016) }\end{array}$} \\
\hline & $k_{1}=2.4 \times 10^{4} \mathrm{M}^{-1} \mathrm{~s}^{-1}$ & & \\
\hline & $k_{2}=3.7 \times 10^{5} \times \mathrm{e}^{(-5530 \times(1 / T-1 / 298))} \mathrm{M}^{-1} \mathrm{~s}^{-1}$ & & \\
\hline & $k_{3}=1.5 \times 10^{9} \times \mathrm{e}^{(-5280 \times(1 / T-1 / 298))} \mathrm{M}^{-1} \mathrm{~s}^{-1}$ & & \\
\hline & $\log _{10}\left(\frac{k}{k^{I_{\mathrm{s}}}=0}\right)=b_{1}\left(\frac{\sqrt{I_{\mathrm{s}}}}{1+\sqrt{I_{\mathrm{s}}}}-0.3 I_{\mathrm{s}}\right)$ & $I_{\mathrm{s}, \max }=0.94 \mathrm{M}$ & \multirow[t]{2}{*}{ Maahs (1983) } \\
\hline & $b_{1}$ is in range of 0.7 to $1.3^{\mathrm{b}}$ & & \\
\hline & $\frac{k}{k^{I_{\mathrm{s}}=0}}=1+b_{2} I_{\mathrm{s}}$ & $I_{\mathrm{s}, \max }=1.2 \mathrm{M}$ & \multirow{2}{*}{$\begin{array}{l}\text { Lagrange et al. } \\
(1993)\end{array}$} \\
\hline & $b_{2}$ is in range of 1.34 to $6.13^{\mathrm{b}}$ & & \\
\hline \multirow[t]{5}{*}{$\mathrm{H}_{2} \mathrm{O}_{2}$} & $k_{4}\left[\mathrm{H}^{+}\right]\left[\mathrm{HSO}_{3}{ }^{-}\right]\left[\mathrm{H}_{2} \mathrm{O}_{2}(\mathrm{aq})\right] /\left(1+K\left[\mathrm{H}^{+}\right]\right)$ & & McArdle \\
\hline & $k_{4}=7.45 \times 10^{7} \times \mathrm{e}^{(-4430 \times(1 / T-1 / 298))} \mathrm{M}^{-1} \mathrm{~s}^{-1}$ & & \multirow[t]{2}{*}{ Hoffmann (1983) } \\
\hline & $K=13 \mathrm{M}^{-1}$ & & \\
\hline & $\log _{10}\left(\frac{\mathrm{k}}{\mathrm{k}^{I_{\mathrm{S}}=0}}\right)=0.36 I_{\mathrm{s}}-\frac{1.018 \sqrt{I_{\mathrm{s}}}}{1+0.17 \sqrt{I_{\mathrm{S}}}}$ & $I_{\mathrm{s}, \max }=5 \mathrm{M}$ & Maaß et al. (1999) \\
\hline & $\ln \left(\frac{k}{k^{I_{S}=0}}\right)=30.374-\frac{6824.2068}{215.365+I_{\mathrm{S}}} \mathrm{c}$ & & Liu et al. (2020) \\
\hline \multirow[t]{5}{*}{$\mathrm{NO}_{2}$} & $k_{5}[\mathrm{~S}(\mathrm{IV})]\left[\mathrm{NO}_{2}(\mathrm{aq})\right]^{\mathrm{d}}$ & & \multirow{3}{*}{$\begin{array}{l}\text { Clifton et al. } \\
(1988) ; \text { Lee and } \\
\text { Schwartz (1983) }\end{array}$} \\
\hline & $k_{5 \text { low }}=2 \times 10^{6} \mathrm{M}^{-1} \mathrm{~s}^{-1}$ & & \\
\hline & $k_{5 \text { high }}=(1.24-2.95) \times 10^{7} \mathrm{M}^{-1} \mathrm{~s}^{-1}$ & & \\
\hline & $\log _{10}\left(\frac{k}{k^{I}=0}\right)=b_{3} I_{\mathrm{s}}$ & Theoretical prediction & \multirow[t]{2}{*}{ Cheng et al. (2016b) } \\
\hline & $b_{3}>0^{\mathrm{e}}$ & & \\
\hline \multirow[t]{6}{*}{$\mathrm{TMI}+\mathrm{O}_{2}{ }^{\mathrm{f}}$} & $k_{6}\left[\mathrm{H}^{+}\right]^{-0.74}[\mathrm{~S}(\mathrm{IV})][\mathrm{Mn}(\mathrm{II})][\mathrm{Fe}(\mathrm{III})](\mathrm{pH} \leq 4.2)$ & & Ibusuki \\
\hline & $k_{6}=3.72 \times 10^{7} \times \mathrm{e}^{(-8431.6 \times(1 / T-1 / 297))} \mathrm{M}^{-2} \mathrm{~s}^{-1}$ & & \multirow[t]{3}{*}{ Takeuchi (1987) } \\
\hline & $k_{7}\left[\mathrm{H}^{+}\right]^{0.67}[\mathrm{~S}(\mathrm{IV})][\mathrm{Mn}(\mathrm{II})][\mathrm{Fe}(\mathrm{III})](\mathrm{pH}>4.2)$ & & \\
\hline & $k_{7}=2.51 \times 10^{13} \times \mathrm{e}^{(-8431.6 \times(1 / T-1 / 297))} \mathrm{M}^{-2} \mathrm{~s}^{-1}$ & & \\
\hline & $\log _{10}\left(\frac{k}{k^{I_{S}=0}}\right)=\frac{b_{4} \sqrt{I_{S}}}{1+\sqrt{I_{S}}} \mathrm{~g}$ & $I_{\mathrm{s}, \max }=2 \mathrm{M}$ & \multirow[t]{2}{*}{$\begin{array}{l}\text { Martin and Hill } \\
(1987,1967)\end{array}$} \\
\hline & $b_{4}$ is in range of -4 to -2 & & \\
\hline
\end{tabular}




\begin{tabular}{lll}
\hline Mn surface & $k_{8} \times f\left(H^{+}\right) \times f(T) \times f\left(I_{s}\right) \times[\mathrm{Mn}(\mathrm{II})] \times\left[\mathrm{SO}_{2}(\mathrm{~g})\right] \times \mathrm{A}$ & Wang et al. (2020) \\
& $k_{8}=11079.30 \quad \mu \mathrm{g} / \mathrm{m}^{3} / \mathrm{min}, \quad \mathrm{A}$ is the surface area \\
& concentration in $\mathrm{nm}^{2} / \mathrm{cm}^{3}$, and the $\mathrm{SO}_{2}$ mixing ratio is in \\
& $\mathrm{ppbV}$ \\
& $f\left(H^{+}\right)=-1 /\left(1-8.83 \times 10^{17} \times\left[\mathrm{H}^{+}\right]-7.84 \times 10^{21} \times\left[\mathrm{H}^{+}\right]\right)$ & \\
& $f(T)=\mathrm{e}^{-11576.08 \times(1 / \mathrm{T}-1 / 298)}$ \\
& $f\left(I_{S}\right)=\left\{\begin{array}{c}1, I_{S}<1.52911 \times 10^{-41} \times \mathrm{e}^{2999.19}+13.8704 \\
10.3, I_{S} \geq 1.52911 \times 10^{-41} \times \mathrm{e}^{\frac{\mathrm{T}}{2999.19}}+13.8704\end{array}\right.$ \\
Nitrate & $1.64 \times \mathrm{EF} \times\left[\mathrm{NO}_{3}^{-}\right] \times \mathrm{J}_{\mathrm{HNO} 3} \times \frac{\mathrm{K}_{\mathrm{HONO}}}{K_{\mathrm{HONO}}+\left[H^{+}\right]} \times v \times \mathrm{A} \times 0.25 \times\left[\mathrm{SO}_{2}\right]$ & Zheng et al. $(2020)$ \\
photolysis & $\mathrm{KHONO}=10^{-3.3} \mathrm{M}, \mathrm{EF}=1 \sim 100$
\end{tabular}

a The aerosol $\mathrm{pH}$ was in the range of 4.1 to 5.2 based on the calculations of ISORRPIA II model in winter and 3.8 to 4.9 in summer, which are consistent with the observed in NCP (Liu et al., 2017;Guo et al., 2017).

b The values of $b_{1}$ and $b_{2}$ are different for different solutions (Maahs, 1983; Lagrange et al., 1994). Since these values vary largely and have a significant impact on the estimated reaction rate at high $I_{\mathrm{s}}$ in aerosol water, we used a medium value of

$130 b_{1}=1.0$ and $b_{2}=1.94$ in according to the calculation in Cheng to show the general pattern. Due to the low concentration of $\mathrm{O}_{3}$ during haze periods, the pathway provides little contribution in the sulfate formation.

${ }^{\mathrm{c}}$ The last expression is the fitting results from the measurement results of Liu et al. (2020) Figure S10. The typical enhancement factor of proton-catalyzed sulfate formation rate is 40 in the haze periods of the winter campaign with an averaged ionic strength as $36.3 \mathrm{M}$.

$135{ }^{\mathrm{d}}$ The $k_{5 \text { low }}$ was taken from Lee and Schwartz (1983). They reported a lower-limit value of $k_{5 \text { low }}=2 \times 10^{6} \mathrm{M}^{-1} \mathrm{~s}^{-1}$ at $\mathrm{pH}$ of 5.8 and 6.4. The $k_{5 h i g h}$ is in the $\mathrm{pH}$ range of 5.3-13 as reported by Clifton et al. (1988) and it increases with increasing $\mathrm{pH}$. We used the value $k_{5 \text { high }}=1.24 \times 10^{7} \mathrm{M}^{-1} \mathrm{~s}^{-1}$ for $\mathrm{pH}<5.3$, and $k_{5 \text { high }}=(0.1239 \mathrm{pH}+0.5954) \times 10^{7} \mathrm{M}^{-1} \mathrm{~s}^{-1}$ for $\mathrm{pH}$ in $5.3-7.8$ in the present calculations.

${ }^{\text {e }}$ The $b_{3}$ was predicted theoretically to be positive as 0.5 (Cheng et al., 2016b), however, no specific value of $b_{3}$ was determined

140 from laboratory work. Because of the high value of ionic strength during the PKU campaign, we proposed an expression for the activity coefficient of $\mathrm{NO}_{2}$ based on the Sechenov theory to reflect the trend of its reaction rate increasing with the concentrations and chose $b 3$ value as 0.01 , typical value of activity enhancement of $\mathrm{NO}_{2}$ with $\mathrm{S}(\mathrm{IV})$ is 2.31 with averaged ionic strength equaling to 36.3 during the haze periods. With $b 3$ equaling to 0.5 proposed in Cheng et al. (2016b), however, typical value is $1.41 \times 10^{18}$.

145 Thus we didn't consider the influence of $I_{\mathrm{S}}$ on $\mathrm{S}(\mathrm{IV})+\mathrm{NO}_{2}$ in aerosol water in our calculation.

${ }^{\mathrm{f}} \mathrm{S}(\mathrm{IV})$ oxidation by $\mathrm{O}_{2}$, which is via a radical chain mechanism, can be initiated by transition metal ions (TMIs) in bulk water ( $k_{6}$ and $k_{7}$ ). In our calculation, the reaction rate $k_{6}$ and $k_{7}$ was used for sulfate production via $\mathrm{S}(\mathrm{IV})+\mathrm{O}_{2}$. Only $\mathrm{Fe}$ (III) and Mn (II) are considered here, since other transition metal ions (TMIs), such as Sc (III), Ti (III), V(III), Cr (III), Co (II), Ni (II), Cu 
(II) and Zn (II), showed much less catalytic activities (Huss Jr et al., 1982). In addition, it has been concluded that the decreased 150 temperature would generally lead to a decrease in overall reaction rate. Based on the measurement results of Ibusuki and Takeuchi (1987), the correction of temperature are considered in the present study.

${ }^{\mathrm{g}}$ Impact of ionic strength on the sulfate formation rate of aqueous phase TMI-catalyzed oxidation of dissolved $\mathrm{SO}_{2}$ by $\mathrm{O}_{2}$ in aerosol particles was studied by Liu et al. (2020). In their results, $b_{4}$ is -3.02 based on the fitting modeling and in the range of -2 for Fe(III) and -4 for Mn(II) (Martin and Hill, 1987, 1967). We used $b_{4}$ as -3.02 in the calculations in the present study. 
Table S3. Calculations of Henry' law coefficients and influence of ionic strength.

\begin{tabular}{|c|c|c|c|}
\hline Gas species & $\begin{array}{l}\text { Henry's law coefficient and the influence of } \\
I_{\mathrm{s}}\end{array}$ & Notes & References \\
\hline \multirow[t]{2}{*}{$\mathrm{SO}_{2}$} & $H_{\mathrm{SO}_{2}}^{I_{\mathrm{s}}=0}=1.23 \times e^{\left(3145.3 \times\left(\frac{1}{T}-\frac{1}{298}\right)\right)}$ & & $\begin{array}{l}\text { Seinfeld and Pandis } \\
(2016)\end{array}$ \\
\hline & $\log _{10}\left(\frac{H_{\mathrm{SO}_{2}}}{H_{\mathrm{SO}_{2}} I_{\mathrm{S}}=0}\right)=\left(\frac{22.3}{T}-0.0997\right) \times I_{\mathrm{S}}$ & $I_{\mathrm{s}, \max }=6 \mathrm{M}$ & Millero et al. (1989) \\
\hline $\mathrm{O}_{3}$ & $H_{\mathrm{O}_{3}}=e^{\left(\frac{2297}{T}-2.659 \times I_{\mathrm{S}}+688 \times \frac{I_{\mathrm{S}}}{T}-12.19\right)}$ & $I_{\mathrm{s}, \max }=0.6 \mathrm{M}$ & $\begin{array}{l}\text { Kosak-Channing and } \\
\text { Helz (1983) }\end{array}$ \\
\hline \multirow[t]{2}{*}{$\mathrm{H}_{2} \mathrm{O}_{2}{ }^{b}$} & $H_{\mathrm{H}_{2} \mathrm{O}_{2}}^{I_{\mathrm{S}}=0}=1.3 \times 10^{5} \times e^{\left(7297.1 \times\left(\frac{1}{T}-\frac{1}{292}\right)\right)}$ & & $\begin{array}{l}\text { Seinfeld and Pandis } \\
(2016) \text {;Chung et al. } \\
(2005)\end{array}$ \\
\hline & $\frac{H_{\mathrm{H}_{2} \mathrm{O}_{2}}}{H_{\mathrm{H}_{2} \mathrm{O}_{2}}^{I_{2}}}=1-1.414 \times 10^{-3} I_{s}^{2}+0.121 I_{s}$ & $I_{\mathrm{s}, \max }=5 \mathrm{M}$ & Liu et al. (2020) \\
\hline $\mathrm{NO}_{2}{ }^{\mathrm{a}}$ & $H_{\mathrm{NO}_{2}}^{I_{\mathrm{s}}=0}=1.0 \times 10^{-2} \times e^{\left(2516.2 \times\left(\frac{1}{T}-\frac{1}{298}\right)\right)}$ & & $\begin{array}{l}\text { Seinfeld and Pandis } \\
(2016)\end{array}$ \\
\hline
\end{tabular}

${ }^{a}$ We didn't consider the influence of $I_{\mathrm{s}}$ on $H_{\mathrm{NO} 2}$ in our calculation due to the lack of relevant laboratory data. $H$ is in unit of M $\mathrm{atm}^{-1}$. 
160 Table S4. Typical activity coefficient values and expressions used in the MARK model

\begin{tabular}{ll}
\hline Species & Calculation expression or typical value \\
\hline Inorganic ions & \\
\hline $\mathrm{H}^{+}$ & 0.4 \\
$\mathrm{OH}^{-}$ & 0.5 \\
$\mathrm{NH}_{4}{ }^{+}$ & 0.2 \\
$\mathrm{Na}^{+}$ & 0.3 \\
$\mathrm{SO}_{4}^{2-}$ & 0.02 \\
$\mathrm{HSO}_{4}{ }^{-}$ & 1 \\
$\mathrm{NO}_{3}{ }^{-}$ & 0.4 \\
$\mathrm{Fe}(\mathrm{II}), \mathrm{Cu}(\mathrm{I}), \mathrm{Cu}(\mathrm{II}), \mathrm{Mn}(\mathrm{II})$ ions and & \\
their hydroxides & \\
$\mathrm{Fe}(\mathrm{III})$ and its hydroxides & 0.001 \\
\hline $\mathrm{Organic}$ matters and Fe-complex & \\
\hline $\mathrm{H}_{2} \mathrm{C}_{2} \mathrm{O}_{4}$ & 0.6 \\
$\mathrm{HC}_{2} \mathrm{O}_{4}^{-}$ & 0.05 \\
$\mathrm{C}_{2} \mathrm{O}_{4}{ }^{2-}$ & 0.43 \\
{$\left[\mathrm{Fe}\left(\mathrm{C}_{2} \mathrm{O}_{4}\right)_{2}\right]^{-}$} & 0.43 \\
{$\left[\mathrm{Fe}\left(\mathrm{C}_{2} \mathrm{O}_{4}\right)\right]^{+}$} & 0.43 \\
{$\left[\mathrm{Fe}\left(\mathrm{C}_{2} \mathrm{O}_{4}\right)_{3}\right]^{3-}$} & 0.001
\end{tabular}

${ }^{a}$ Non-ideality is treated with the approach by Zuend et al. (2008);Zuend et al. (2011) applied in the AIOMFAC model (Aerosol Inorganic-Organic Mixtures Functional groups Activity Coefficients, http://www.aiomfac.caltech.edu/index.html, last access: 18 July 2020). 
165 Table S5. Kinetic data for the simulation of reactions in the aerosol particle condensed phase.

\begin{tabular}{|c|c|c|c|}
\hline Number & Reaction & $k_{298}\left(\mathrm{M}^{-\mathrm{n}+1} \mathrm{~s}^{-1}\right)$ & $E a / R(\mathrm{~K})$ \\
\hline \multicolumn{4}{|c|}{ Iron reactions } \\
\hline A1 & $\mathrm{Fe}^{2+}+\mathrm{H}_{2} \mathrm{O}_{2(\mathrm{a})} \rightarrow \mathrm{Fe}^{3+}+\mathrm{OH}_{(\mathrm{a})}+\mathrm{OH}^{-}$ & 70 & 5050 \\
\hline $\mathrm{A} 2$ & $\mathrm{Fe}^{2+}+\mathrm{O}_{3(\mathrm{a})} \rightarrow \mathrm{FeO}^{2+}+\mathrm{O}_{2(\mathrm{a})}$ & $8.2 \times 10^{5}$ & 4690 \\
\hline A3 & $\mathrm{FeO}^{2+}+\mathrm{H}_{2} \mathrm{O}_{2(\mathrm{a})} \rightarrow \mathrm{Fe}^{3+}+\mathrm{HO}_{2(\mathrm{a})}+\mathrm{OH}^{-}$ & $9.5 \times 10^{3}$ & 2766 \\
\hline A4 & $\mathrm{FeO}^{2+}+\mathrm{HO}_{2(\mathrm{a})} \rightarrow \mathrm{Fe}^{3+}+\mathrm{O}_{2(\mathrm{a})}+\mathrm{OH}^{-}$ & $2 \times 10^{6}$ & 0 \\
\hline A5 & $\mathrm{FeO}^{2+}+\mathrm{OH}_{(\mathrm{a})}+\mathrm{H}^{+} \rightarrow \mathrm{Fe}^{3+}+\mathrm{H}_{2} \mathrm{O}_{2(\mathrm{a})}$ & $1 \times 10^{7}$ & 0 \\
\hline A6 & $\mathrm{FeO}^{2+}+\mathrm{H}_{2} \mathrm{O}_{(\mathrm{a})} \rightarrow \mathrm{Fe}^{3+}+\mathrm{OH}_{(\mathrm{a})}+\mathrm{OH}^{-}$ & $2.3 \times 10^{-2}$ & 4100 \\
\hline A7 & $\mathrm{FeO}^{2+}+\mathrm{Fe}^{2+}+\mathrm{H}_{2} \mathrm{O}_{(\mathrm{a})} \rightarrow 2 \mathrm{Fe}^{3+}+2 \mathrm{OH}^{+}$ & $7.2 \times 10^{4}$ & 842 \\
\hline A8 & $\mathrm{FeO}^{2+}+\mathrm{Fe}^{2+}+\mathrm{H}_{2} \mathrm{O}_{(\mathrm{a})} \rightarrow \mathrm{Fe}(\mathrm{OH})_{2} \mathrm{Fe}^{4+}$ & $1.8 \times 10^{4}$ & 5052 \\
\hline A9 & $\mathrm{Fe}(\mathrm{OH})_{2} \mathrm{Fe}^{4+}+2 \mathrm{H}^{+} \rightarrow 2 \mathrm{Fe}^{3+}+2 \mathrm{H}_{2} \mathrm{O}_{(\mathrm{a})}$ & 2 & 5653 \\
\hline A10 & $\mathrm{Fe}(\mathrm{OH})_{2} \mathrm{Fe}^{4+} \rightarrow 2 \mathrm{Fe}^{3+}+2 \mathrm{OH}^{-}$ & 0.49 & 8780 \\
\hline A11 & $\mathrm{FeO}^{2+}+\mathrm{HNO}_{2(\mathrm{a})} \rightarrow \mathrm{Fe}^{3+}+\mathrm{NO}_{2(\mathrm{a})}+\mathrm{OH}^{-}$ & $1.1 \times 10^{4}$ & 4150 \\
\hline $\mathrm{A} 12$ & $\mathrm{FeO}^{2+}+\mathrm{H}^{+}+\mathrm{NO}_{2}^{-} \rightarrow \mathrm{Fe}^{3+}+\mathrm{NO}_{2(\mathrm{a})}+\mathrm{OH}^{-}$ & $2.5 \times 10^{5}$ & 0 \\
\hline A13 & $\mathrm{FeO}^{2+}+\mathrm{HSO}_{3}^{-} \rightarrow \mathrm{Fe}^{3+}+\mathrm{OH}^{-}+\mathrm{SO}_{3}^{-}$ & $1 \times 10^{5}$ & 0 \\
\hline A14 & $\mathrm{Fe}^{2+}+\mathrm{OH}_{(\mathrm{a})} \rightarrow \mathrm{Fe}(\mathrm{OH})^{2+}$ & $4.3 \times 10^{8}$ & 1100 \\
\hline A15 & $\mathrm{Fe}(\mathrm{OH})^{2+}+\mathrm{HO}_{2(\mathrm{a})} \rightarrow \mathrm{Fe}^{2+}+\mathrm{O}_{2(\mathrm{a})}+\mathrm{H}_{2} \mathrm{O}_{(\mathrm{a})}$ & $1.3 \times 10^{5}$ & 0 \\
\hline A16 & $\mathrm{Fe}(\mathrm{OH})^{2+}+\mathrm{O}_{2}^{-} \rightarrow \mathrm{Fe}^{2+}+\mathrm{O}_{2(\mathrm{a})}+\mathrm{OH}^{-}$ & $1.5 \times 10^{8}$ & 0 \\
\hline A17 & $\mathrm{Fe}^{3+}+\mathrm{O}_{2}^{-} \rightarrow \mathrm{Fe}^{2+}+\mathrm{O}_{2(\mathrm{a})}$ & $1.5 \times 10^{8}$ & 0 \\
\hline A18 & $\mathrm{Fe}^{2+}+2 \mathrm{H}^{+}+\mathrm{O}_{2}^{-} \rightarrow \mathrm{Fe}^{3+}+\mathrm{H}_{2} \mathrm{O}_{2(\mathrm{a})}$ & $1 \times 10^{7}$ & 0 \\
\hline A19 & $\mathrm{Fe}^{2+}+\mathrm{HO}_{2(\mathrm{a})}+\mathrm{H}^{+} \rightarrow \mathrm{Fe}^{3+}+\mathrm{H}_{2} \mathrm{O}_{2(\mathrm{a})}$ & $1.2 \times 10^{6}$ & 5050 \\
\hline A20 & $\mathrm{Fe}(\mathrm{OH})_{2}^{+}+\mathrm{O}_{2}^{-} \rightarrow \mathrm{Fe}^{2+}+\mathrm{O}_{2(\mathrm{a})}+2 \mathrm{OH}^{-}$ & $1.5 \times 10^{8}$ & 0 \\
\hline A 21 & $\mathrm{Fe}(\mathrm{OH})^{2+}+\mathrm{HSO}_{3}^{-} \rightarrow \mathrm{Fe}^{2+}+\mathrm{SO}_{3}^{-}+\mathrm{H}_{2} \mathrm{O}_{(\mathrm{a})}$ & 30 & 0 \\
\hline A 22 & $\mathrm{Fe}^{2+}+\mathrm{SO}_{5}^{-}+\mathrm{H}_{2} \mathrm{O}_{(\mathrm{a})} \rightarrow \mathrm{Fe}(\mathrm{OH})^{2+}+\mathrm{HSO}_{5}^{-}$ & $2.65 \times 10^{7}$ & 5809 \\
\hline A23 & $\mathrm{Fe}^{2+}+\mathrm{HSO}_{5}^{-} \rightarrow \mathrm{Fe}(\mathrm{OH})^{2+}+\mathrm{SO}_{4}^{-}$ & $3 \times 10^{4}$ & 0 \\
\hline A24 & $\mathrm{Fe}^{2+}+\mathrm{SO}_{4}^{-} \rightarrow \mathrm{Fe}^{3+}+\mathrm{SO}_{4}^{2-}$ & $4.6 \times 10^{9}$ & -2165 \\
\hline A 25 & $\mathrm{Fe}^{2+}+\mathrm{S}_{2} \mathrm{O}_{8}^{2-} \rightarrow \mathrm{Fe}^{3+}+\mathrm{SO}_{4}^{-}+\mathrm{SO}_{4}^{2-}$ & 17 & 0 \\
\hline \multicolumn{4}{|c|}{ Copper reactions } \\
\hline A26 & $\mathrm{Cu}^{+}+2 \mathrm{H}^{+}+\mathrm{O}_{2}^{-} \rightarrow \mathrm{Cu}^{2+}+\mathrm{H}_{2} \mathrm{O}_{2(\mathrm{a})}$ & $8 \times 10^{9}$ & 0 \\
\hline
\end{tabular}




\begin{tabular}{|c|c|c|c|}
\hline A27 & $\mathrm{Cu}^{+}+\mathrm{HO}_{2(\mathrm{a})}+\mathrm{H}^{+} \rightarrow \mathrm{Cu}^{2+}+\mathrm{H}_{2} \mathrm{O}_{2(\mathrm{a})}$ & $2.2 \times 10^{9}$ & 0 \\
\hline A28 & $\mathrm{Cu}^{+}+\mathrm{OH}_{(\mathrm{a})} \rightarrow \mathrm{Cu}^{2+}+\mathrm{OH}^{-}$ & $3 \times 10^{9}$ & 0 \\
\hline A29 & $\mathrm{Cu}^{2+}+\mathrm{HO}_{2(\mathrm{a})} \rightarrow \mathrm{Cu}^{+}+\mathrm{H}^{+}+\mathrm{O}_{2(\mathrm{a})}$ & $1 \times 10^{8}$ & 0 \\
\hline $\mathrm{A} 30$ & $\mathrm{Cu}^{2+}+\mathrm{O}_{2}^{-} \rightarrow \mathrm{Cu}^{+}+\mathrm{O}_{2(\mathrm{a})}$ & $1 \times 10^{9}$ & 0 \\
\hline A31 & $\mathrm{Cu}^{+}+\mathrm{O}_{2(\mathrm{a})} \rightarrow \mathrm{Cu}^{2+}+\mathrm{O}_{2}^{-}$ & $4.6 \times 10^{5}$ & 0 \\
\hline A32 & $\mathrm{Cu}^{+}+\mathrm{H}^{+}+\mathrm{O}_{3(\mathrm{a})} \rightarrow \mathrm{Cu}^{2+}+\mathrm{O}_{2(\mathrm{a})}+\mathrm{OH}_{(\mathrm{a})}$ & $3 \times 10^{7}$ & 0 \\
\hline A33 & $\mathrm{Cu}^{+}+\mathrm{H}_{2} \mathrm{O}_{2(\mathrm{a})} \rightarrow \mathrm{Cu}^{2+}+\mathrm{OH}_{(\mathrm{a})}+\mathrm{OH}^{-}$ & $7 \times 10^{3}$ & 0 \\
\hline A34 & $\mathrm{Cu}^{+}+\mathrm{SO}_{4}^{-} \rightarrow \mathrm{Cu}^{2+}+\mathrm{SO}_{4}^{2-}$ & $3 \times 10^{8}$ & 0 \\
\hline \multicolumn{4}{|c|}{ Mn reactions } \\
\hline A35 & $\mathrm{Mn}^{4+}+\mathrm{H}_{2} \mathrm{O}_{2(\mathrm{a})} \rightarrow \mathrm{Mn}^{2+}+\mathrm{O}_{2(\mathrm{a})}+2 \mathrm{H}^{+}$ & $7.3 \times 10^{4}$ & 0 \\
\hline A36 & $\mathrm{Mn}^{3+}+\mathrm{H}_{2} \mathrm{O}_{2(\mathrm{a})} \rightarrow \mathrm{Mn}^{2+}+\mathrm{HO}_{2(\mathrm{a})}+\mathrm{H}^{+}$ & $7.3 \times 10^{4}$ & 0 \\
\hline A37 & $\mathrm{MnOH}^{2+}+\mathrm{H}_{2} \mathrm{O}_{2(\mathrm{a})} \rightarrow \mathrm{MnO}_{2}^{+}+\mathrm{H}^{+}$ & $2.8 \times 10^{3}$ & 0 \\
\hline A 38 & $\mathrm{MnO}_{2}^{+}+\mathrm{HO}_{2(\mathrm{a})}+\mathrm{H}^{+} \rightarrow \mathrm{H}_{2} \mathrm{O}_{2(\mathrm{a})}+\mathrm{Mn}^{2+}+\mathrm{O}_{2(\mathrm{a})}$ & $1 \times 10^{7}$ & 0 \\
\hline A39 & $\mathrm{Mn}^{2+}+\mathrm{OH}_{(\mathrm{a})} \rightarrow \mathrm{Mn}^{3+}+\mathrm{OH}^{-}$ & $3.4 \times 10^{7}$ & 0 \\
\hline A40 & $2 \mathrm{MnO}_{2}^{+}+2 \mathrm{H}^{+} \rightarrow 2 \mathrm{Mn}^{2+}+\mathrm{H}_{2} \mathrm{O}_{2(\mathrm{a})}$ & $6 \times 10^{6}$ & 0 \\
\hline A41 & $\mathrm{MnO}^{2+}+2 \mathrm{H}^{+}+\mathrm{Mn}^{2+} \rightarrow 2 \mathrm{Mn}^{3+}$ & $1 \times 10^{5}$ & 0 \\
\hline A 42 & $\mathrm{Mn}^{2+}+\mathrm{O}_{3(\mathrm{a})}+\mathrm{H}^{+} \rightarrow \mathrm{Mn}^{3+}+\mathrm{O}_{2(\mathrm{a})}+\mathrm{OH}_{(\mathrm{a})}$ & $1.65 \times 10^{5}$ & 0 \\
\hline A43 & $\mathrm{Mn}^{2+}+\mathrm{NO}_{3(\mathrm{a})} \rightarrow \mathrm{Mn}^{3+}+\mathrm{NO}_{3}^{-}$ & $1.5 \times 10^{6}$ & 0 \\
\hline A44 & $\mathrm{Mn}^{2+}+\mathrm{HSO}_{5}^{-} \rightarrow \mathrm{Mn}^{3+}+\mathrm{SO}_{4}^{-}+\mathrm{OH}^{-}$ & $3 \times 10^{4}$ & 0 \\
\hline A45 & $\mathrm{Mn}^{2+}+\mathrm{SO}_{5}^{-} \rightarrow \mathrm{Mn}^{3+}+\mathrm{HSO}_{5}^{-}+\mathrm{OH}^{-}$ & $1 \times 10^{10}$ & 0 \\
\hline A46 & $\mathrm{Mn}^{2+}+\mathrm{SO}_{4}^{-} \rightarrow \mathrm{Mn}^{3+}+\mathrm{SO}_{4}^{2-}$ & $1.4 \times 10^{7}$ & 4089 \\
\hline A47 & $\mathrm{MnHSO}_{3}^{+}+\mathrm{Mn}^{3+} \rightarrow \mathrm{H}^{+}+2 \mathrm{Mn}^{2+}+\mathrm{SO}_{3}^{-}$ & $1.3 \times 10^{6}$ & 0 \\
\hline \multicolumn{4}{|c|}{$\mathrm{Cu}-\mathrm{Fe}-\mathrm{Mn}$ redox reactions } \\
\hline A48 & $\mathrm{Cu}^{+}+\mathrm{Fe}^{3+} \rightarrow \mathrm{Cu}^{2+}+\mathrm{Fe}^{2+}$ & $1.3 \times 10^{7}$ & 0 \\
\hline A49 & $\mathrm{Cu}^{+}+\mathrm{FeOH}^{2+} \rightarrow \mathrm{Cu}^{2+}+\mathrm{Fe}^{2+}+\mathrm{OH}^{-}$ & $1.3 \times 10^{7}$ & 0 \\
\hline A50 & $\mathrm{Cu}^{+}+\mathrm{Fe}(\mathrm{OH})_{2}^{+} \rightarrow \mathrm{Cu}^{2+}+\mathrm{Fe}^{2+}+2 \mathrm{OH}^{-}$ & $1.3 \times 10^{7}$ & 0 \\
\hline A51 & $\mathrm{Mn}^{3+}+\mathrm{Fe}^{2+} \rightarrow \mathrm{Mn}^{2+}+\mathrm{Fe}^{3+}$ & $1.6 \times 10^{4}$ & 0 \\
\hline A52 & $\mathrm{Mn}^{2+}+\mathrm{FeO}^{2+}+2 \mathrm{H}^{+} \rightarrow \mathrm{Mn}^{3+}+\mathrm{Fe}^{3+}$ & $1 \times 10^{4}$ & 2562 \\
\hline \multicolumn{4}{|c|}{ Hydroxide redox reactions } \\
\hline A53 & $\mathrm{O}_{2}^{-}+\mathrm{O}_{3(\mathrm{a})} \rightarrow \mathrm{O}_{2(\mathrm{a})}+\mathrm{O}_{3}^{-}$ & $1.5 \times 10^{9}$ & 2200 \\
\hline
\end{tabular}




\begin{tabular}{|c|c|c|c|}
\hline A54 & $2 \mathrm{HO}_{2(\mathrm{a})} \rightarrow \mathrm{H}_{2} \mathrm{O}_{2(\mathrm{a})}+\mathrm{O}_{2(\mathrm{a})}$ & $8.3 \times 10^{5}$ & 2700 \\
\hline A55 & $\mathrm{HO}_{2(\mathrm{a})}+\mathrm{O}_{2}^{-}+\mathrm{H}_{2} \mathrm{O}_{(\mathrm{a})} \rightarrow \mathrm{H}_{2} \mathrm{O}_{2(\mathrm{a})}+\mathrm{O}_{2(\mathrm{a})}+\mathrm{OH}^{-}$ & $9.7 \times 10^{7}$ & 1060 \\
\hline A56 & $\mathrm{HO}_{2(\mathrm{a})}+\mathrm{OH}_{(\mathrm{a})} \rightarrow \mathrm{O}_{2(\mathrm{a})}+\mathrm{H}_{2} \mathrm{O}_{(\mathrm{a})}$ & $1 \times 10^{10}$ & 0 \\
\hline A57 & $\mathrm{O}_{2}^{-}+\mathrm{OH}_{(\mathrm{a})} \rightarrow \mathrm{O}_{2(\mathrm{a})}+\mathrm{OH}^{-}$ & $1.1 \times 10^{10}$ & 2120 \\
\hline A58 & $\mathrm{H}_{2} \mathrm{O}_{2(\mathrm{a})}+\mathrm{OH}_{(\mathrm{a})} \rightarrow \mathrm{HO}_{2(\mathrm{a})}+\mathrm{H}_{2} \mathrm{O}_{(\mathrm{a})}$ & $3 \times 10^{7}$ & 1680 \\
\hline \multicolumn{4}{|c|}{ Organic reactions } \\
\hline A59 & $\mathrm{H}_{2} \mathrm{C}_{2} \mathrm{O}_{4}+\mathrm{OH}_{(\mathrm{a})} \rightarrow \mathrm{H}_{2} \mathrm{O}_{(\mathrm{a})}+\mathrm{C}_{2} \mathrm{O}_{4}^{-}+\mathrm{H}^{+}$ & $1.9 \times 10^{8}$ & 2800 \\
\hline A60 & $\mathrm{C}_{2} \mathrm{O}_{4}^{2-}+\mathrm{OH}_{(\mathrm{a})} \rightarrow \mathrm{OH}^{-}+\mathrm{C}_{2} \mathrm{O}_{4}^{-}$ & $1.6 \times 10^{8}$ & 4300 \\
\hline A61 & $\mathrm{C}_{2} \mathrm{O}_{4}^{-}+\mathrm{O}_{2(\mathrm{a})} \rightarrow 2 \mathrm{CO}_{2(\mathrm{a})}+\mathrm{O}_{2}^{-}$ & $2 \times 10^{9}$ & 2800 \\
\hline A62 & $\mathrm{HC}_{2} \mathrm{O}_{4}^{-}+\mathrm{SO}_{5}^{-} \rightarrow \mathrm{C}_{2} \mathrm{O}_{4}^{-}+\mathrm{HSO}_{5}^{-}$ & $5 \times 10^{3}$ & 0 \\
\hline A63 & $\mathrm{HC}_{2} \mathrm{O}_{4}^{-}+\mathrm{SO}_{4}^{-} \rightarrow \mathrm{C}_{2} \mathrm{O}_{4}^{-}+\mathrm{H}^{+}+\mathrm{SO}_{4}^{2-}$ & $3.35 \times 10^{5}$ & 0 \\
\hline A64 & $\mathrm{HC}_{2} \mathrm{O}_{4}^{-}+\mathrm{NO}_{3(\mathrm{a})} \rightarrow \mathrm{C}_{2} \mathrm{O}_{4}^{-}+\mathrm{H}^{+}+\mathrm{NO}_{3}^{-}$ & $6.8 \times 10^{7}$ & 0 \\
\hline A65 & $\mathrm{C}_{2} \mathrm{O}_{4}^{2-}+\mathrm{H}^{+}+\mathrm{SO}_{5}^{-} \rightarrow \mathrm{C}_{2} \mathrm{O}_{4}^{-}+\mathrm{HSO}_{5}^{-}$ & $1 \times 10^{4}$ & 0 \\
\hline A66 & $\mathrm{C}_{2} \mathrm{O}_{4}^{2-}+\mathrm{SO}_{4}^{-} \rightarrow \mathrm{C}_{2} \mathrm{O}_{4}^{-}+\mathrm{SO}_{4}^{2-}$ & $1.05 \times 10^{6}$ & 0 \\
\hline A67 & $\mathrm{C}_{2} \mathrm{O}_{4}^{2-}+\mathrm{NO}_{3(\mathrm{a})} \rightarrow \mathrm{C}_{2} \mathrm{O}_{4}^{-}+\mathrm{NO}_{3}^{-}$ & $2.2 \times 10^{8}$ & 0 \\
\hline A68 & $\mathrm{HCOOH}+\mathrm{OH}\left(+\mathrm{O}_{2}\right) \rightarrow$ Products & $3.2 \times 10^{9}$ & 0 \\
\hline \multicolumn{4}{|c|}{ Fe-oxalate complex reactions } \\
\hline A69 & $\mathrm{Fe}^{2+}+\mathrm{C}_{2} \mathrm{O}_{4}^{2-} \rightarrow \mathrm{FeC}_{2} \mathrm{O}_{4(\mathrm{a})}$ & $1 \times 10^{6}$ & 0 \\
\hline A70 & $\mathrm{FeC}_{2} \mathrm{O}_{4(\mathrm{a})} \rightarrow \mathrm{Fe}^{2+}+\mathrm{C}_{2} \mathrm{O}_{4}^{2-}$ & $1 \times 10^{3}$ & 0 \\
\hline A71 & $\mathrm{FeC}_{2} \mathrm{O}_{4}^{+}+\mathrm{O}_{2}^{-} \rightarrow \mathrm{FeC}_{2} \mathrm{O}_{4(\mathrm{a})}+\mathrm{O}_{2(\mathrm{a})}$ & $1 \times 10^{6}$ & 0 \\
\hline A72 & $\mathrm{FeC}_{2} \mathrm{O}_{4}^{+}+\mathrm{HO}_{2(\mathrm{a})} \rightarrow \mathrm{FeC}_{2} \mathrm{O}_{4(\mathrm{a})}+\mathrm{O}_{2(\mathrm{a})}+\mathrm{H}^{+}$ & $1.2 \times 10^{5}$ & 0 \\
\hline \multicolumn{4}{|c|}{ Sulfur and Nitrate compound reactions } \\
\hline A73 & $\mathrm{HSO}_{3}^{-}+\mathrm{OH}_{(\mathrm{a})} \rightarrow \mathrm{SO}_{3}^{-}+\mathrm{H}_{2} \mathrm{O}_{(\mathrm{a})}$ & $2.7 \times 10^{9}$ & 0 \\
\hline A74 & $\mathrm{OH}_{(\mathrm{a})}+\mathrm{SO}_{3}^{2-} \rightarrow \mathrm{OH}^{-}+\mathrm{SO}_{3}^{-}$ & $4.6 \times 10^{9}$ & 0 \\
\hline A75 & $\mathrm{H}_{2} \mathrm{O}_{(\mathrm{a})}+\mathrm{N}_{2} \mathrm{O}_{5(\mathrm{a})} \rightarrow 2 \mathrm{H}^{+}+2 \mathrm{NO}_{3}^{-}$ & $5 \times 10^{9}$ & 0 \\
\hline A76 & $\mathrm{N}_{2} \mathrm{O}_{5(\mathrm{a})} \rightarrow \mathrm{NO}_{2}^{+}+\mathrm{NO}_{3}^{-}$ & $1 \times 10^{9}$ & 0 \\
\hline A77 & $\mathrm{H}_{2} \mathrm{O}_{(\mathrm{a})}+\mathrm{NO}_{2}^{+} \rightarrow 2 \mathrm{H}^{+}+\mathrm{NO}_{3}^{-}$ & $8.9 \times 10^{7}$ & 0 \\
\hline A78 & $\mathrm{Fe}^{2+}+\mathrm{NO}_{3(\mathrm{a})} \rightarrow \mathrm{Fe}^{3+}+\mathrm{NO}_{3}^{-}$ & $8 \times 10^{6}$ & 0 \\
\hline A79 & $\mathrm{H}_{2} \mathrm{O}_{2(\mathrm{a})}+\mathrm{NO}_{3(\mathrm{a})} \rightarrow \mathrm{HO}_{2(\mathrm{a})}+\mathrm{H}^{+}+\mathrm{NO}_{3}^{-}$ & $4.9 \times 10^{6}$ & 2000 \\
\hline $\mathrm{A} 80$ & $\mathrm{HO}_{2(\mathrm{a})}+\mathrm{NO}_{3(\mathrm{a})} \rightarrow \mathrm{H}^{+}+\mathrm{NO}_{3}^{-}+\mathrm{O}_{2(\mathrm{a})}$ & $3 \times 10^{9}$ & 0 \\
\hline
\end{tabular}




\begin{tabular}{|c|c|c|c|}
\hline A81 & $\mathrm{NO}_{3(\mathrm{a})}+\mathrm{O}_{2}^{-} \rightarrow \mathrm{NO}_{3}^{-}+\mathrm{O}_{2(\mathrm{a})}$ & $3 \times 10^{9}$ & 0 \\
\hline A82 & $\mathrm{HSO}_{3}^{-}+\mathrm{NO}_{3(\mathrm{a})} \rightarrow \mathrm{H}^{+}+\mathrm{NO}_{3}^{-}+\mathrm{SO}_{3}^{-}$ & $1.3 \times 10^{9}$ & 2000 \\
\hline A83 & $\mathrm{NO}_{3(\mathrm{a})}+\mathrm{SO}_{3}^{2-} \rightarrow \mathrm{NO}_{3}^{-}+\mathrm{SO}_{3}^{-}$ & $3 \times 10^{8}$ & 0 \\
\hline A84 & $\mathrm{HSO}_{4}^{-}+\mathrm{NO}_{3(\mathrm{a})} \rightarrow \mathrm{H}^{+}+\mathrm{NO}_{3}^{-}+\mathrm{SO}_{4}^{-}$ & $2.6 \times 10^{5}$ & 0 \\
\hline A85 & $\mathrm{NO}_{3(\mathrm{a})}+\mathrm{SO}_{4}^{2-} \rightarrow \mathrm{NO}_{3}^{-}+\mathrm{SO}_{4}^{-}$ & $1 \times 10^{5}$ & 0 \\
\hline A86 & $\mathrm{NO}_{2(\mathrm{a})}+\mathrm{OH}_{(\mathrm{a})} \rightarrow \mathrm{HOONO}_{(\mathrm{a})}$ & $1.2 \times 10^{10}$ & 0 \\
\hline A87 & $\mathrm{NO}_{2(\mathrm{a})}+\mathrm{O}_{2}^{-} \rightarrow \mathrm{NO}_{2}^{-}+\mathrm{O}_{2(\mathrm{a})}$ & $1 \times 10^{8}$ & 0 \\
\hline A 88 & $2 \mathrm{NO}_{2(\mathrm{a})}+\mathrm{H}_{2} \mathrm{O}_{(\mathrm{a})} \rightarrow \mathrm{HNO}_{2(\mathrm{a})}+\mathrm{H}^{+}+\mathrm{NO}_{3}^{-}$ & $8.4 \times 10^{7}$ & -2900 \\
\hline A89 & $\mathrm{NO}_{2}^{-}+\mathrm{OH}_{(\mathrm{a})} \rightarrow \mathrm{NO}_{2(\mathrm{a})}+\mathrm{OH}^{-}$ & $9.1 \times 10^{9}$ & 0 \\
\hline A90 & $\mathrm{NO}_{2}^{-}+\mathrm{SO}_{4}^{-} \rightarrow \mathrm{NO}_{2(\mathrm{a})}+\mathrm{SO}_{4}^{2-}$ & $7.2 \times 10^{8}$ & 0 \\
\hline A91 & $\mathrm{NO}_{2}^{-}+\mathrm{NO}_{3(\mathrm{a})} \rightarrow \mathrm{NO}_{2(\mathrm{a})}+\mathrm{NO}_{3}^{-}$ & $1.4 \times 10^{9}$ & 0 \\
\hline A92 & $\mathrm{NO}_{2}^{-}+\mathrm{O}_{3(\mathrm{a})} \rightarrow \mathrm{NO}_{3}^{-}+\mathrm{O}_{2(\mathrm{a})}$ & $5 \times 10^{5}$ & 6900 \\
\hline A93 & $\mathrm{HNO}_{2(\mathrm{a})}+\mathrm{OH}_{(\mathrm{a})} \rightarrow \mathrm{NO}_{2(\mathrm{a})}+\mathrm{H}_{2} \mathrm{O}_{(\mathrm{a})}$ & $1.1 \times 10^{10}$ & 0 \\
\hline A94 & $\mathrm{HNO}_{4(\mathrm{a})}+\mathrm{HSO}_{3}^{-} \rightarrow \mathrm{HSO}_{4}^{-}+\mathrm{H}^{+}+\mathrm{NO}_{3}^{-}$ & $3.3 \times 10^{5}$ & 0 \\
\hline A95 & $\mathrm{H}_{2} \mathrm{O}_{(\mathrm{a})}+\mathrm{SO}_{3(\mathrm{a})} \rightarrow 2 \mathrm{H}^{+}+\mathrm{SO}_{4}^{2-}$ & $1 \times 10^{10}$ & 0 \\
\hline A96 & $\mathrm{O}_{3(\mathrm{a})}+\mathrm{SO}_{3}^{2-} \rightarrow \mathrm{O}_{2(\mathrm{a})}+\mathrm{SO}_{4}^{2-}$ & $1.5 \times 10^{9}$ & 5280 \\
\hline A97 & $2 \mathrm{SO}_{5}^{-} \rightarrow \mathrm{O}_{2(\mathrm{a})}+\mathrm{S}_{2} \mathrm{O}_{8}^{2-}$ & $4.8 \times 10^{7}$ & 2600 \\
\hline A98 & $2 \mathrm{SO}_{5}^{-} \rightarrow \mathrm{O}_{2(\mathrm{a})}+2 \mathrm{SO}_{4}^{-}$ & $2.2 \times 10^{8}$ & 2600 \\
\hline A99 & $\mathrm{H}^{+}+\mathrm{O}_{2}^{-}+\mathrm{SO}_{5}^{-} \rightarrow \mathrm{HSO}_{5}^{-}+\mathrm{O}_{2(\mathrm{a})}$ & $2.34 \times 10^{8}$ & 0 \\
\hline A100 & $\mathrm{O}_{2(\mathrm{a})}+\mathrm{SO}_{3(\mathrm{a})} \rightarrow \mathrm{SO}_{5}^{-}$ & $2.5 \times 10^{9}$ & 0 \\
\hline A101 & $\mathrm{HSO}_{3}^{-}+\mathrm{SO}_{5}^{-} \rightarrow \mathrm{HSO}_{5}^{-}+\mathrm{SO}_{3}^{-}$ & $8.6 \times 10^{3}$ & 0 \\
\hline A102 & $\mathrm{HSO}_{3}^{-}+\mathrm{SO}_{5}^{-} \rightarrow \mathrm{H}^{+}+\mathrm{SO}_{4}^{-}+\mathrm{SO}_{4}^{2-}$ & $3.6 \times 10^{2}$ & 0 \\
\hline A103 & $\mathrm{H}^{+}+\mathrm{SO}_{3}^{2-}+\mathrm{SO}_{5}^{-} \rightarrow \mathrm{HSO}_{5}^{-}+\mathrm{SO}_{3}^{-}$ & $2.1 \times 10^{5}$ & 0 \\
\hline A104 & $\mathrm{SO}_{3}^{2-}+\mathrm{SO}_{5}^{-} \rightarrow \mathrm{SO}_{4}^{-}+\mathrm{SO}_{4}^{2-}$ & $5.5 \times 10^{5}$ & 0 \\
\hline A105 & $\mathrm{HSO}_{4}^{-}+\mathrm{OH}_{(\mathrm{a})} \rightarrow \mathrm{SO}_{4}^{-}+\mathrm{H}_{2} \mathrm{O}_{(\mathrm{a})}$ & $3.5 \times 10^{5}$ & 0 \\
\hline A106 & $2 \mathrm{SO}_{4}^{-} \rightarrow \mathrm{S}_{2} \mathrm{O}_{8}^{2-}$ & $6.1 \times 10^{8}$ & 840 \\
\hline A107 & $\mathrm{HSO}_{3}^{-}+\mathrm{SO}_{4}^{-} \rightarrow \mathrm{H}^{+}+\mathrm{SO}_{3}^{-}+\mathrm{SO}_{4}^{2-}$ & $5.8 \times 10^{8}$ & 0 \\
\hline A108 & $\mathrm{SO}_{3}^{2-}+\mathrm{SO}_{4}^{-} \rightarrow \mathrm{SO}_{3}^{-}+\mathrm{SO}_{4}^{2-}$ & $3.4 \times 10^{8}$ & 1200 \\
\hline A109 & $\mathrm{H}_{2} \mathrm{O}_{2(\mathrm{a})}+\mathrm{SO}_{4}^{-} \rightarrow \mathrm{HO}_{2(\mathrm{a})}+\mathrm{H}^{+}+\mathrm{SO}_{4}^{2-}$ & $1.7 \times 10^{7}$ & 0 \\
\hline A110 & $\mathrm{HO}_{2(\mathrm{a})}+\mathrm{SO}_{4}^{-} \rightarrow \mathrm{H}^{+}+\mathrm{O}_{2(\mathrm{a})}+\mathrm{SO}_{4}^{2-}$ & $3.5 \times 10^{9}$ & 0 \\
\hline
\end{tabular}




\begin{tabular}{lcll}
\hline $\mathrm{A} 111$ & $\mathrm{O}_{2}^{-}+\mathrm{SO}_{4}^{-} \rightarrow \mathrm{O}_{2(\mathrm{a})}+\mathrm{SO}_{4}^{2-}$ & $3.5 \times 10^{9}$ & 0 \\
$\mathrm{~A} 112$ & $\mathrm{NO}_{3}^{-}+\mathrm{SO}_{4}^{-} \rightarrow \mathrm{NO}_{3(\mathrm{a})}+\mathrm{SO}_{4}^{2-}$ & $5 \times 10^{4}$ & 0 \\
$\mathrm{~A} 113$ & $\mathrm{OH}^{-}+\mathrm{SO}_{4}^{-} \rightarrow \mathrm{OH}_{(\mathrm{a})}+\mathrm{SO}_{4}^{2-}$ & $1.4 \times 10^{7}$ & 0 \\
$\mathrm{~A} 114$ & $\mathrm{H}_{2} \mathrm{O}_{(\mathrm{a})}+\mathrm{SO}_{4}^{-} \rightarrow \mathrm{H}^{+}+\mathrm{OH}_{(\mathrm{a})}+\mathrm{SO}_{4}^{2-}$ & 11 & 1100 \\
$\mathrm{~A} 115$ & $\mathrm{HSO}_{3}^{-}+\mathrm{HSO}_{5}^{-}+\mathrm{H}^{+} \rightarrow 3 \mathrm{H}^{+}+2 \mathrm{SO}_{4}^{2-}$ & $7.14 \times 10^{6}$ & 0 \\
$\mathrm{~A} 116$ & $\mathrm{SO}_{3}^{2-}+\mathrm{HSO}_{5}^{-}+\mathrm{H}^{+} \rightarrow 2 \mathrm{H}^{+}+2 \mathrm{SO}_{4}^{2-}$ & $7.14 \times 10^{6}$ & 0 \\
$\mathrm{~A} 117$ & $\mathrm{HSO}_{5}^{-}+\mathrm{OH}_{(\mathrm{a})} \rightarrow \mathrm{SO}_{5}^{-}+\mathrm{H}_{2} \mathrm{O}_{(\mathrm{a})}$ & $1.7 \times 10^{7}$ & 0 \\
$\mathrm{~A} 118$ & $\mathrm{OH}_{(\mathrm{a})}+\mathrm{SO}_{4}^{-} \rightarrow \mathrm{HSO}_{5}^{-}$ & $1 \times 10^{10}$ & 0 \\
$\mathrm{~A} 119$ & $\mathrm{H}_{2} \mathrm{O}_{2(\mathrm{a})}+\mathrm{HSO}_{3}^{-}+\mathrm{H}^{+} \rightarrow 2 \mathrm{H}^{+}+\mathrm{SO}_{4}^{2-}+\mathrm{H}_{2} \mathrm{O}_{(\mathrm{a})}$ & $7.2 \times 10^{7}$ & 4000 \\
$\mathrm{~A} 120$ & $\mathrm{O}_{3(\mathrm{a})}+\mathrm{SO}_{2(\mathrm{a})}+\mathrm{H}_{2} \mathrm{O}_{(\mathrm{a})} \rightarrow \mathrm{HSO}_{4}^{-}+\mathrm{H}^{+}+\mathrm{O}_{2(\mathrm{a})}$ & $2.4 \times 10^{4}$ & 0 \\
$\mathrm{~A} 121$ & $\mathrm{HSO}_{3}^{-}+\mathrm{O}_{3(\mathrm{a})} \rightarrow \mathrm{SO}_{4}^{2-}+\mathrm{H}^{+}+\mathrm{O}_{2(\mathrm{a})}$ & $3.7 \times 10^{5}$ & 5530 \\
$\mathrm{~A} 122$ & $\mathrm{NO}_{3(\mathrm{a})}+\mathrm{OH}^{-} \rightarrow \mathrm{NO}_{3}^{-}+\mathrm{OH}_{(\mathrm{a})}$ & $9.4 \times 10^{7}$ & 2700 \\
\hline
\end{tabular}


Table S6. Photolysis rates (aqueous phase) used in the model at noon $\left(\mathrm{sza}=20^{\circ}\right)$

\begin{tabular}{lrl}
\hline Number & Reaction & $\mathrm{J}_{0}\left(\mathrm{~s}^{-1}\right)$ \\
\hline J1 & $\mathrm{H}_{2} \mathrm{O}_{2(\mathrm{a})}+h v \rightarrow 2 \mathrm{OH}_{(\mathrm{a})}$ & $6.98 \times 10^{-6}$ \\
$\mathrm{~J} 2$ & $\mathrm{Fe}^{3+}+\mathrm{H}_{2} \mathrm{O}_{(\mathrm{a})}+h v \rightarrow \mathrm{Fe}^{2+}+\mathrm{OH}_{(\mathrm{a})}+\mathrm{H}^{+}$ & $9.3 \times 10^{-6}$ \\
$\mathrm{~J} 3$ & $\mathrm{Fe}(\mathrm{OH})^{2+}+h v \rightarrow \mathrm{Fe}^{2+}+\mathrm{OH}_{(\mathrm{a})}$ & $4.39 \times 10^{-3}$ \\
J4 & $\mathrm{Fe}(\mathrm{OH})_{2}^{+}+h v \rightarrow \mathrm{Fe}^{2+}+\mathrm{OH}_{(\mathrm{a})}+\mathrm{OH}^{-}$ & $5.63 \times 10^{-3}$ \\
$\mathrm{~J} 5$ & $\mathrm{NO}_{2}^{-}+h v \rightarrow \mathrm{NO}_{(\mathrm{a})}+\mathrm{OH}_{(\mathrm{a})}$ & $2.51 \times 10^{-5}$ \\
$\mathrm{~J} 6$ & $\mathrm{NO}_{3}^{-}+h v \rightarrow \mathrm{NO}_{2(\mathrm{a})}+\mathrm{OH}_{(\mathrm{a})}$ & $4.15 \times 10^{-7}$ \\
J7 & $\mathrm{Fe}\left[\left(\mathrm{C}_{2} \mathrm{O}_{4}\right)_{2}\right]^{-}+h v \rightarrow \mathrm{C}_{2} \mathrm{O}_{4}^{2-}+\mathrm{C}_{2} \mathrm{O}_{4}^{-}+\mathrm{Fe}^{2+}$ & $2.30 \times 10^{-2}$ \\
J8 & $\mathrm{Fe}\left[\left(\mathrm{C}_{2} \mathrm{O}_{4}\right)_{3}\right]^{3-}+h v \rightarrow 2 \mathrm{C}_{2} \mathrm{O}_{4}^{2-}+\mathrm{C}_{2} \mathrm{O}_{4}^{-}+\mathrm{Fe}^{2+}$ & $5.76 \times 10^{-2}$ \\
J9 & $\mathrm{FeC}_{2} \mathrm{O}_{4}^{+}+h v \rightarrow \mathrm{Fe}^{2+}+\mathrm{C}_{2} \mathrm{O}_{4}^{-}$ & $7.20 \times 10^{-4}$ \\
$\mathrm{~J} 10$ & $\mathrm{NO}_{3(\mathrm{a})}+h v \rightarrow \mathrm{NO}_{(\mathrm{a})}+\mathrm{O}_{2(\mathrm{a})}$ & $2.32 \times 10^{-2 *}$ \\
$\mathrm{~J} 11$ & $\mathrm{NO}_{3(\mathrm{a})}+h v \rightarrow \mathrm{NO}_{2(\mathrm{a})}+\mathrm{O}_{(\mathrm{a})}^{3 \mathrm{p}}$ & $2.01 \times 10^{-1 *}$ \\
\hline
\end{tabular}

*Estimated as in the gas phase 
Table S7. Aqueous equilibrium reactions

\begin{tabular}{|c|c|c|c|c|c|c|}
\hline Number & Reaction & $K_{298}(\mathrm{M})$ & $\begin{array}{l}k_{298}\left(\mathrm{M}^{-\mathrm{n}} \mathrm{s}^{-1}\right) \\
\text { forward }\end{array}$ & $E_{d} / R(\mathrm{~K})$ & $\begin{array}{l}k_{298}\left(\mathrm{M}^{-\mathrm{n}} \mathrm{s}^{-1}\right) \\
\text { backward }\end{array}$ & $\begin{array}{l}E_{a} / R \\
(\mathrm{~K})\end{array}$ \\
\hline E1 & $\mathrm{H}_{2} \mathrm{O}_{(\mathrm{a})} \leftrightarrow \mathrm{H}^{+}+\mathrm{OH}^{-}$ & $1.8 \times 10^{-16}$ & $2.34 \times 10^{-5}$ & 6800 & $1.3 \times 10^{11}$ & 0 \\
\hline E2 & $\mathrm{NH}_{3(\mathrm{a})}+\mathrm{H}_{2} \mathrm{O}_{(\mathrm{a})} \leftrightarrow \mathrm{NH}_{4}^{+}+\mathrm{OH}^{-}$ & $1.17 \times 10^{-5}$ & $6.02 \times 10^{5}$ & 560 & $3.4 \times 10^{10}$ & \\
\hline E3 & $\mathrm{HO}_{2(\mathrm{a})} \leftrightarrow \mathrm{H}^{+}+\mathrm{O}_{2}^{-}$ & $1.6 \times 10^{-5}$ & $8.0 \times 10^{5}$ & 0 & $5 \times 10^{10}$ & 0 \\
\hline E4 & $\mathrm{HNO}_{3(\mathrm{a})} \leftrightarrow \mathrm{H}^{+}+\mathrm{NO}_{3}^{-}$ & 22 & $1.1 \times 10^{12}$ & -1800 & $5 \times 10^{10}$ & \\
\hline E5 & $\mathrm{HNO}_{2(\mathrm{a})} \leftrightarrow \mathrm{H}^{+}+\mathrm{NO}_{2}^{-}$ & $5.30 \times 10^{-4}$ & $2.65 \times 10^{7}$ & 1760 & $5 \times 10^{10}$ & \\
\hline E6 & $\mathrm{HNO}_{4(\mathrm{a})} \leftrightarrow \mathrm{H}^{+}+\mathrm{O}_{2} \mathrm{NO}_{2}^{-}$ & $1 \times 10^{-5}$ & $5 \times 10^{5}$ & & $5 \times 10^{10}$ & \\
\hline E7 & $\mathrm{HO}_{2(\mathrm{a})}+\mathrm{NO}_{2(\mathrm{a})} \leftrightarrow \mathrm{HNO}_{4(\mathrm{a})}$ & $2.17 \times 10^{9}$ & $1 \times 10^{7}$ & & $4.6 \times 10^{-3}$ & \\
\hline E8 & $\mathrm{HO}_{2(\mathrm{a})}+\mathrm{SO}_{2(\mathrm{a})} \leftrightarrow \mathrm{HSO}_{3}^{-}+\mathrm{H}^{+}$ & $3.14 \times 10^{-4}$ & $6.27 \times 10^{4}$ & -1940 & $2.0 \times 10^{8}$ & \\
\hline E9 & $\mathrm{HSO}_{3}^{-} \leftrightarrow \mathrm{H}^{+}+\mathrm{SO}_{3}^{2-}$ & $6.22 \times 10^{-8}$ & 3110 & -1960 & $5 \times 10^{10}$ & \\
\hline E10 & $\mathrm{H}_{2} \mathrm{SO}_{4(\mathrm{a})} \leftrightarrow \mathrm{HSO}_{4}^{-}+\mathrm{H}^{+}$ & $1 \times 10^{3}$ & $5 \times 10^{13}$ & & $5 \times 10^{10}$ & \\
\hline E11 & $\mathrm{HSO}_{4}^{-} \leftrightarrow \mathrm{H}^{+}+\mathrm{SO}_{4}^{2-}$ & $1.02 \times 10^{-2}$ & $1.02 \times 10^{9}$ & -2700 & $1 \times 10^{11}$ & \\
\hline E12 & $\mathrm{Fe}^{3+}+\mathrm{H}_{2} \mathrm{O}_{(\mathrm{a})} \leftrightarrow \mathrm{Fe}(\mathrm{OH})^{2+}+\mathrm{H}^{+}$ & $1.09 \times 10^{-4}$ & $4.7 \times 10^{4}$ & & $4.3 \times 10^{8}$ & \\
\hline E13 & $\mathrm{Fe}(\mathrm{OH})^{2+}+\mathrm{H}_{2} \mathrm{O}_{(\mathrm{a})} \leftrightarrow \mathrm{Fe}(\mathrm{OH})_{2}^{+}+\mathrm{H}^{+}$ & $1.38 \times 10^{-7}$ & $1.1 \times 10^{3}$ & & $8 \times 10^{9}$ & \\
\hline E14 & $\mathrm{Fe}^{3+}+\mathrm{SO}_{4}^{2-} \leftrightarrow \mathrm{Fe}\left(\mathrm{SO}_{4}\right)^{+}$ & $1.78 \times 10^{-2}$ & $3.2 \times 10^{3}$ & & $1.8 \times 10^{5}$ & \\
\hline E15 & $\mathrm{Cu}^{2+}+\mathrm{OH}_{(\mathrm{a})} \leftrightarrow \mathrm{Cu}(\mathrm{OH})^{2+}$ & $1.17 \times 10^{4}$ & $3.5 \times 10^{8}$ & & $3 \times 10^{4}$ & \\
\hline E16 & $\mathrm{HO}_{3(\mathrm{a})} \leftrightarrow \mathrm{H}^{+}+\mathrm{O}_{3}^{-}$ & $5 \times 10^{-9}$ & 330 & & $5.2 \times 10^{10}$ & \\
\hline E17 & $\mathrm{HOONO}_{(\mathrm{a})} \leftrightarrow \mathrm{H}^{+}+\mathrm{OONO}^{-}$ & $1 \times 10^{-6}$ & $5 \times 10^{4}$ & & $5 \times 10^{10}$ & \\
\hline E18 & $\mathrm{Fe}\left(\mathrm{C}_{2} \mathrm{O}_{4}\right)^{+} \leftrightarrow \mathrm{C}_{2} \mathrm{O}_{4}^{2-}+\mathrm{Fe}^{3+}$ & $4.0 \times 10^{-10}$ & $3 \times 10^{-3}$ & & $7.5 \times 10^{6}$ & \\
\hline E19 & $\mathrm{Fe}\left(\mathrm{C}_{2} \mathrm{O}_{4}\right)_{2}^{-} \leftrightarrow \mathrm{C}_{2} \mathrm{O}_{4}^{2-}+\mathrm{Fe}\left(\mathrm{C}_{2} \mathrm{O}_{4}\right)^{+}$ & $1.59 \times 10^{-7}$ & $3 \times 10^{-3}$ & & $1.89 \times 10^{4}$ & \\
\hline E20 & $\mathrm{Fe}\left(\mathrm{C}_{2} \mathrm{O}_{4}\right)_{3}^{3-} \leftrightarrow \mathrm{C}_{2} \mathrm{O}_{4}^{2-}+\mathrm{Fe}\left(\mathrm{C}_{2} \mathrm{O}_{4}\right)_{2}^{-}$ & $2.65 \times 10^{-5}$ & $3 \times 10^{-3}$ & & 114 & \\
\hline
\end{tabular}


Table S8. Kinetic data for the simulation of gas-liquid phase conversion reactions

\begin{tabular}{|c|c|c|c|}
\hline Number & Reaction* & $\begin{array}{l}k_{298}\left(\mathrm{M}^{-\mathrm{n}+1} \mathrm{~s}^{-1}\right) \\
\text { forward }\end{array}$ & $\begin{array}{l}k_{298} \quad\left(\mathrm{M}^{-\mathrm{n}+1} \quad \mathrm{~s}^{-1}\right) \\
\text { backward }\end{array}$ \\
\hline $\mathrm{T} 1$ & $\mathrm{CO}_{2(\mathrm{~g})} \leftrightarrow \mathrm{CO}_{2(\mathrm{a})}$ & $k_{m t} \mathrm{CO} \times \mathrm{ALWC}$ & $k_{m t} \mathrm{CO}_{2} /\left(\mathrm{H}_{\mathrm{CO}} \mathrm{RT}\right)$ \\
\hline $\mathrm{T} 2$ & $\mathrm{NH}_{3(\mathrm{~g})} \leftrightarrow \mathrm{NH}_{3(\mathrm{a})}$ & $k_{m t N H 3} \times \mathrm{ALWC}$ & $k_{m t N H 3} /\left(H_{N H 3} \mathrm{RT}\right)$ \\
\hline $\mathrm{T} 3$ & $\mathrm{O}_{3(\mathrm{~g})} \leftrightarrow \mathrm{O}_{3(\mathrm{a})}$ & $k_{m t}{ }_{03} \times \mathrm{ALWC}$ & $k_{m t} \mathrm{O}_{3} /\left(\mathrm{H}_{O 3} \mathrm{RT}\right)$ \\
\hline $\mathrm{T} 4$ & $\mathrm{HO}_{2(\mathrm{~g})} \leftrightarrow \mathrm{HO}_{2(\mathrm{a})}$ & $k_{m t} \mathrm{HO}_{2} \times \mathrm{ALWC}$ & $k_{m t} \mathrm{HO}_{2} /\left(\mathrm{H}_{\mathrm{HO} 2} \mathrm{RT}\right)$ \\
\hline T5 & $\mathrm{OH}_{(\mathrm{g})} \leftrightarrow \mathrm{OH}_{(\mathrm{a})}$ & $k_{m t} \mathrm{OH} \times \mathrm{ALWC}$ & $k_{m t}$ OH$/\left(H_{O H} \mathrm{RT}\right)$ \\
\hline $\mathrm{T} 6$ & $\mathrm{H}_{2} \mathrm{O}_{2(\mathrm{~g})} \leftrightarrow \mathrm{H}_{2} \mathrm{O}_{2(\mathrm{a})}$ & $k_{m t} \mathrm{H}_{2 \mathrm{O} 2} \times \mathrm{ALWC}$ & 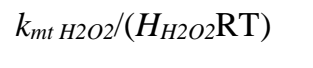 \\
\hline $\mathrm{T} 7$ & $\mathrm{NO}_{3(\mathrm{~g})} \leftrightarrow \mathrm{NO}_{3(\mathrm{a})}$ & $k_{m t} \mathrm{NO}_{3} \times \mathrm{ALWC}$ & 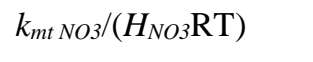 \\
\hline $\mathrm{T} 8$ & $\mathrm{~N}_{2} \mathrm{O}_{5(\mathrm{~g})} \leftrightarrow \mathrm{N}_{2} \mathrm{O}_{5(\mathrm{a})}$ & $k_{m t N 2 O 5} \times \mathrm{ALWC}$ & $k_{m t ~ N 2 O 5} /\left(H_{N 2 O 5} \mathrm{RT}\right)$ \\
\hline T9 & $\mathrm{NO}_{2(\mathrm{~g})} \leftrightarrow \mathrm{NO}_{2(\mathrm{a})}$ & 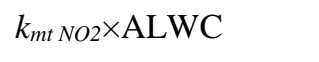 & 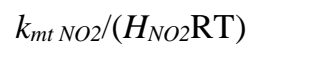 \\
\hline $\mathrm{T} 10$ & $\mathrm{SO}_{2(\mathrm{~g})} \leftrightarrow \mathrm{SO}_{2(\mathrm{a})}$ & $k_{m t S O} \times \mathrm{ALWC}$ & 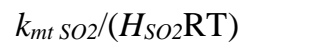 \\
\hline
\end{tabular}

$175 * \mathrm{k}_{\mathrm{mt}}$ is related to the particle diameters and the aerosol liquid water in different diameter bins. For this reason, the mass transfer rates are corrected by the particle 11 bins diameters in the two field campaigns. The rate $\mathrm{k}_{\mathrm{mt}}$ equals to $\sum_{i}^{11} k_{m t_{\_} i} \times L_{i}$ 
Table S9. Concentration of transition metals in $\mathrm{PM}_{2.5}$ in urban areas.

\begin{tabular}{lllllll}
\hline Sampling site & Period & Method & $\mathrm{Fe}$ & $\mathrm{Mn}$ & $\mathrm{Cu}$ & References \\
\hline China, Beijing, Urban & $2018.8-2019.8$ & XRF & 596 & 27.9 & 7.37 & Zhao et al. (2021) \\
China, Beijing, Urban & $2015.9-2016.1$ & XRF & 686 & 60.2 & 25.1 & Zhang et al. (2019) \\
China, Beijing, Urban & $2016.6-2017.5$ & ED-XRF & 738 & 37 & 32 & Cui et al. (2019) \\
China, Beijing, Urban & $2014.1-10$ & ICP-AES & 1650 & 55 & 108 & Gao et al. (2018) \\
China, Beijing, Urban & $2016.1-2017.5$ & XRF & 629 & 32 & 24 & Cui et al. (2020) \\
$\begin{array}{l}\text { China, Beijing, Urban } \\
\text { China, Zhengzhou, }\end{array}$ & 2016.1 & ICP-AES & 2823 & 92.3 & 48 & Duan et al. (2012) \\
Urban & $2017.10-2018.7$ & XRF & 1361 & 157 & 29.2 & He et al. (2019) \\
China, Nanjing, Urban & $2016.12-$ & & & & & \\
China, Shanghai, Urban & 2017.12 & XRF & 577 & 48.9 & 27.2 & Yu et al. (2019) \\
$\begin{array}{l}\text { Canada, } \quad \text { Hamilton, } \\
\text { Urban }\end{array}$ & $2014.1-2017.6$ & XRF & 49.6 & 0.83 & 2.76 & Sofowote et al. (2019) \\
$\begin{array}{l}\text { India, New Delhi, } \\
\text { Urban }\end{array}$ & $2013.1-2016.12$ & WD-XRF & 780 & 10 & 100 & Jain et al. (2020) \\
\hline
\end{tabular}




\section{SI References}

Ali, H. M., Iedema, M., Yu, X.-Y., and Cowin, J. P.: Ionic strength dependence of the oxidation of SO2 by $\mathrm{H} 2 \mathrm{O} 2$ in sodium chloride particles, Atmospheric Environment, 89, 731-738, 2014.

Beltran, F. J.: Ozone reaction kinetics for water and wastewater systems, crc Press, 2003.

185 Burkholder, J., Sander, S., Abbatt, J., Barker, J., Cappa, C., Crounse, J., Dibble, T., Huie, R., Kolb, C., and Kurylo, M.: Chemical kinetics and photochemical data for use in atmospheric studies; evaluation number 19, Pasadena, CA: Jet Propulsion Laboratory, National Aeronautics and Space ..., 2020.

Chang, Y., Huang, K., Deng, C., Zou, Z., Liu, S., and Zhang, Y.: First long-term and near real-time measurement of atmospheric trace elements in Shanghai, China, Atmos. Chem. Phys. Discuss., in Review, 2017.

190 Cheng, Y., Zheng, G., Wei, C., Mu, Q., Zheng, B., Wang, Z., Gao, M., Zhang, Q., He, K., and Carmichael, G.: Reactive nitrogen chemistry in aerosol water as a source of sulfate during haze events in China, Science Advances, 2, e1601530, 2016a. Cheng, Y., Zheng, G., Wei, C., Mu, Q., Zheng, B., Wang, Z., Gao, M., Zhang, Q., He, K., Carmichael, G., Pöschl, U., and Su, H.: Reactive nitrogen chemistry in aerosol water as a source of sulfate during haze events in China, Science Advances, 2, e1601530, 10.1126/sciadv.1601530, 2016b.

195 Chung, M. Y., Muthana, S., Paluyo, R. N., and Hasson, A. S.: Measurements of effective Henry's law constants for hydrogen peroxide in concentrated salt solutions, Atmospheric Environment, 39, 2981-2989, 2005.

Clegg, S., Kleeman, M., Griffin, R., and Seinfeld, J.: Effects of uncertainties in the thermodynamic properties of aerosol components in an air quality model-Part 1: Treatment of inorganic electrolytes and organic compounds in the condensed phase, Atmospheric Chemistry and Physics, 8, 1027-1085, 2008.

200 Clever, H. L.: Setchenov salt-effect parameter, Journal of Chemical and Engineering Data, 28, 340-343, 1983.

Clifton, C. L., Altstein, N., and Huie, R. E.: Rate constant for the reaction of nitrogen dioxide with sulfur (IV) over the pH range 5.3-13, Environmental science \& technology, 22, 586-589, 1988.

Cui, Y., Ji, D., Chen, H., Gao, M., Maenhaut, W., He, J., and Wang, Y.: Characteristics and sources of hourly trace elements in airborne fine particles in urban Beijing, China, Journal of Geophysical Research: Atmospheres, 124, 11595-11613, 2019.

205 Cui, Y., Ji, D., He, J., Kong, S., and Wang, Y.: In situ continuous observation of hourly elements in PM2. 5 in urban beijing, China: Occurrence levels, temporal variation, potential source regions and health risks, Atmospheric Environment, 222, $117164,2020$.

Duan, J. C., Tan, J. H., Wang, S. L., Hao, J. M., and Chail, F. H.: Size distributions and sources of elements in particulate matter at curbside, urban and rural sites in Beijing, Journal of Environmental Sciences, 24, 87-94, 10.1016/s1001-

$210 \quad 0742(11) 60731-6,2012$.

Fountoukis, C., and Nenes, A.: ISORROPIA II: a computationally efficient thermodynamic equilibrium model for $\mathrm{K}^{+}-\mathrm{Ca}^{2+}-$ $\mathrm{Mg}^{2+}-\mathrm{NH}_{4}{ }^{+}-\mathrm{Na}^{+}-\mathrm{SO}_{4}{ }^{2-}-\mathrm{NO}_{3}{ }^{-}-\mathrm{Cl}^{-}-\mathrm{H} 2 \mathrm{O}$ aerosols, Atmospheric Chemistry and Physics, 7, 4639-4659, $2007 \mathrm{a}$.

Fountoukis, C., and Nenes, A.: ISORROPIA II: a computationally efficient thermodynamic equilibrium model for $\mathrm{K}^{+}-\mathrm{Ca}^{2+}$ $\mathrm{Mg}^{2+}-\mathrm{NH}_{4}{ }^{+}-\mathrm{Na}^{+}-\mathrm{SO}_{4}{ }^{2-}-\mathrm{NO}_{3}{ }^{-}-\mathrm{Cl}^{-}-\mathrm{H} 2 \mathrm{O}$ aerosols, Atmospheric Chemistry and Physics Discussions, 7, 1893-1939, $2007 \mathrm{~b}$.

215 Gao, J., Wang, K., Wang, Y., Liu, S., Zhu, C., Hao, J., Liu, H., Hua, S., and Tian, H.: Temporal-spatial characteristics and source apportionment of PM2. 5 as well as its associated chemical species in the Beijing-Tianjin-Hebei region of China, Environmental Pollution, 233, 714-724, 2018.

Guo, H., Weber, R. J., and Nenes, A.: High levels of ammonia do not raise fine particle pH sufficiently to yield nitrogen oxidedominated sulfate production, Scientific Reports, 7, 12109, 10.1038/s41598-017-11704-0, 2017.

220 He, R.-D., Zhang, Y.-S., Chen, Y.-Y., Jin, M.-J., Han, S.-J., Zhao, J.-S., Zhang, R.-Q., and Yan, Q.-S.: Heavy metal pollution characteristics and ecological and health risk assessment of atmospheric PM2. 5 in a living area of Zhengzhou City, Huan jing ke xue= Huanjing kexue, 40, 4774-4782, 2019.

Herrmann, H., Schaefer, T., Tilgner, A., Styler, S. A., Weller, C., Teich, M., and Otto, T.: Tropospheric Aqueous-Phase Chemistry: Kinetics, Mechanisms, and Its Coupling to a Changing Gas Phase, Chemical Reviews, 115, 4259-4334, 225 10.1021/cr500447k, 2015.

Huss Jr, A., Lim, P. K., and Eckert, C.: Oxidation of aqueous sulfur dioxide. 1. Homogeneous manganese (II) and iron (II) catalysis at low pH, The Journal of Physical Chemistry, 86, 4224-4228, 1982. 
Ibusuki, T., and Takeuchi, K.: Sulfur dioxide oxidation by oxygen catalyzed by mixtures of manganese(II) and iron(III) in aqueous solutions at environmental reaction conditions, Atmospheric Environment (1967), 21, 1555-1560, 230 https://doi.org/10.1016/0004-6981(87)90317-9, 1987.

Jain, S., Sharma, S., Vijayan, N., and Mandal, T.: Seasonal characteristics of aerosols (PM2. 5 and PM10) and their source apportionment using PMF: a four year study over Delhi, India, Environmental Pollution, 262, 114337, 2020.

Kontogeorgis, G. M., Maribo-Mogensen, B., and Thomsen, K.: The Debye-Hückel theory and its importance in modeling electrolyte solutions, Fluid Phase Equilibria, 462, 130-152, 2018.

235 Kosak-Channing, L. F., and Helz, G. R.: Solubility of ozone in aqueous solutions of 0-0.6 M ionic strength at 5-30. degree. C, Environmental science \& technology, 17, 145-149, 1983.

Lagrange, J., Pallares, C., Wenger, G., and Lagrange, P.: Electrolyte effects on aqueous atmospheric oxidation of sulphur dioxide by hydrogen peroxide, Atmospheric Environment. Part A. General Topics, 27, 129-137, https://doi.org/10.1016/09601686(93)90342-V, 1993.

240 Lee, Y., and Schwartz, S. E.: Kinetics of oxidation of aqueous sulfur (IV) by nitrogen dioxide, Precipitation Scavenging, Dry Deposition and Resuspension, 1, 453-470, 1983.

Li, J., Polka, H.-M., and Gmehling, J.: A gE model for single and mixed solvent electrolyte systems: 1. Model and results for strong electrolytes, Fluid Phase Equilibria, 94, 89-114, 1994.

Linder, P. W., and Murray, K.: Correction of formation constants for ionic strength, from only one or two data points: An 245 examination of the use of the extended Debye-Hückel equation, Talanta, 29, 377-382, 1982.

Liu, M., Song, Y., Zhou, T., Xu, Z., Yan, C., Zheng, M., Wu, Z., Hu, M., Wu, Y., and Zhu, T.: Fine particle pH during severe haze episodes in northern China, Geophysical Research Letters, 44, 5213-5221, https://doi.org/10.1002/2017GL073210, 2017. Liu, T., Clegg, S. L., and Abbatt, J. P. D.: Fast oxidation of sulfur dioxide by hydrogen peroxide in deliquesced aerosol particles, Proceedings of the National Academy of Sciences, 117, 1354-1359, 10.1073/pnas.1916401117, 2020.

250 Maahs, H. G.: Kinetics and mechanism of the oxidation of S(IV) by ozone in aqueous solution with particular reference to $\mathrm{SO} 2$ conversion in nonurban tropospheric clouds, Journal of Geophysical Research: Oceans, 88, 10721-10732, https://doi.org/10.1029/JC088iC15p10721, 1983.

Maaß, F., Elias, H., and Wannowius, K. J.: Kinetics of the oxidation of hydrogen sulfite by hydrogen peroxide in aqueous solution:: ionic strength effects and temperature dependence, Atmospheric Environment, 33, 4413-4419, 1999.

255 Martin, L. R., and Hill, M. W.: The iron catalyzed oxidation of sulfur: Reconciliation of the literature rates, Atmospheric Environment (1967), 21, 1487-1490, 1967.

Martin, L. R., and Hill, M. W.: The effect of ionic strength on the manganese catalyzed oxidation of sulfur (IV), Atmospheric Environment (1967), 21, 2267-2270, 1987.

McArdle, J. V., and Hoffmann, M. R.: Kinetics and mechanism of the oxidation of aquated sulfur dioxide by hydrogen peroxide

260 at low pH, The Journal of Physical Chemistry, 87, 5425-5429, 1983.

Millero, F. J., Hershey, J. B., Johnson, G., and Zhang, J.-Z.: The solubility of SO 2 and the dissociation of H 2 SO 3 in NaCl solutions, J Atmos Chem, 8, 377-389, 1989.

Ming, Y., and Russell, L. M.: Thermodynamic equilibrium of organic-electrolyte mixtures in aerosol particles, AIChE Journal, 48, 1331-1348, 2002.

265 Pitzer, K. S.: Ion interaction approach: theory and data correlation, Activity coefficients in electrolyte solutions, 2, 75-153, 1991.

Polka, H.-M., Li, J., and Gmehling, J.: A gE model for single and mixed solvent electrolyte systems: 2. Results and comparison with other models, Fluid phase equilibria, 94, 115-127, 1994.

Raatikainen, T., and Laaksonen, A.: Application of several activity coefficient models to water-organic-electrolyte aerosols of 270 atmospheric interest, 2005.

Rischbieter, E., Stein, H., and Schumpe, A.: Ozone solubilities in water and aqueous salt solutions, Journal of Chemical \& Engineering Data, 45, 338-340, 2000.

Ross, H. B., and Noone, K. J.: A numerical investigation of the destruction of peroxy radical by Cu ion catalyzed-reactions of atmospheric particles, J Atmos Chem, 12, 121-136, 10.1007/bf00115775, 1991.

275 Rusumdar, A., Wolke, R., Tilgner, A., and Herrmann, H.: Treatment of non-ideality in the SPACCIM multiphase model-Part 1: Model development, 2016. 
Rusumdar, A. J., Tilgner, A., Wolke, R., and Herrmann, H.: Treatment of non-ideality in the SPACCIM multiphase model Part 2: Impacts on the multiphase chemical processing in deliquesced aerosol particles, Atmos. Chem. Phys., 20, 10351-10377, 10.5194/acp-20-10351-2020, 2020.

280 Seinfeld, J. H., and Pandis, S. N.: Atmospheric chemistry and physics: from air pollution to climate change, John Wiley \& Sons, 2016.

Sofowote, U. M., Di Federico, L. M., Healy, R. M., Debosz, J., Su, Y., Wang, J., and Munoz, A.: Heavy metals in the nearroad environment: Results of semi-continuous monitoring of ambient particulate matter in the greater Toronto and Hamilton area, Atmospheric Environment: X, 1, 100005, 2019.

285 Wang, X., Gemayel, R., Hayeck, N., Perrier, S., Charbonnel, N., Xu, C., Chen, H., Zhu, C., Zhang, L., Wang, L., Nizkorodov, S. A., Wang, X., Wang, Z., Wang, T., Mellouki, A., Riva, M., Chen, J., and George, C.: Atmospheric Photosensitization: A New Pathway for Sulfate Formation, Environmental Science \& Technology, 54, 3114-3120, 10.1021/acs.est.9b06347, 2020. Yu, Y., He, S., Wu, X., Zhang, C., Yao, Y., Liao, H., Wang, Q. g., and Xie, M.: PM2. 5 elements at an urban site in Yangtze River Delta, China: High time-resolved measurement and the application in source apportionment, Environmental Pollution,

290 253, 1089-1099, 2019.

Zhang, B., Zhou, T., Liu, Y., Yan, C., Li, X., Yu, J., Wang, S., Liu, B., and Zheng, M.: Comparison of water-soluble inorganic ions and trace metals in $\mathrm{PM}_{2.5}$ between online and offline measurements in Beijing during winter, Atmospheric Pollution Research, 10, 1755-1765, https://doi.org/10.1016/j.apr.2019.07.007, 2019.

Zhao, S., Tian, H., Luo, L., Liu, H., Wu, B., Liu, S., Bai, X., Liu, W., Liu, X., Wu, Y., Lin, S., Guo, Z., Lv, Y., and Xue, Y.:

295 Temporal variation characteristics and source apportionment of metal elements in PM2.5 in urban Beijing during 2018-2019, Environmental Pollution, 268, 115856, https://doi.org/10.1016/j.envpol.2020.115856, 2021.

Zheng, H., Song, S., Sarwar, G., Gen, M., Wang, S., Ding, D., Chang, X., Zhang, S., Xing, J., Sun, Y., Ji, D., Chan, C. K., Gao, J., and McElroy, M. B.: Contribution of Particulate Nitrate Photolysis to Heterogeneous Sulfate Formation for Winter Haze in China, Environmental Science \& Technology Letters, 7, 632-638, 10.1021/acs.estlett.0c00368, 2020.

300 Zuend, A., Marcolli, C., Luo, B. P., and Peter, T.: A thermodynamic model of mixed organic-inorganic aerosols to predict activity coefficients, 2008.

Zuend, A., Marcolli, C., Booth, A., Lienhard, D. M., Soonsin, V., Krieger, U., Topping, D. O., McFiggans, G., Peter, T., and Seinfeld, J. H.: New and extended parameterization of the thermodynamic model AIOMFAC: calculation of activity coefficients for organic-inorganic mixtures containing carboxyl, hydroxyl, carbonyl, ether, ester, alkenyl, alkyl, and aromatic functional groups, Atmospheric Chemistry and Physics, 11, 9155-9206, 2011. 